\title{
Turpentine derived secondary amine botanical agrochemicals for crop protection: Synthesis, activity evaluation and QSAR study
}

Shichao $\mathrm{Xu}^{\mathrm{a}, \mathrm{b}^{*}}$, Xiaojing Zeng ${ }^{\mathrm{a}}$, Songlin Dai ${ }^{\mathrm{a}}$, Jing Wang ${ }^{\mathrm{a}, \mathrm{b}}$, Yuxiang Chen ${ }^{\mathrm{a}, \mathrm{b}}$, Yunfei Shic, Xian Cheng ${ }^{\mathrm{a}}$, Shengliang Liao ${ }^{\mathrm{c}^{*}}$, Jie Song ${ }^{\mathrm{d}^{*}}$, Zhendong Zhao ${ }^{\mathrm{a}, \mathrm{b}^{*}}$

anstitute of Chemical Industry of Forest Products, CAF; Key Lab. of Biomass Energy and Material, Jiangsu Province; Co-Innovation Center of Efficient Processing and Utilization of Forest Resources, Jiangsu Province; Key Lab. of Chemical Engineering of Forest Products, National Forestry and Grassland Administration; National Engineering Lab. for Biomass Chemical Utilization, Nanjing 210042, China

${ }^{b}$ Research Institute of Forestry New Technology, CAF, Beijing 100091, China

${ }^{c}$ College of Forestry, Jiangxi Agricultural University; East China Woody Fragrance and Flavor Engineering Research Center of National Forestry and Grassland Administration, Nanchang 330045, China

${ }^{d}$ Department of Chemistry and Biochemistry, University of Michigan-Flint, Flint, MI 48502, United States

*Corresponding author E-mail: zdzhao@189.cn;

jiesong@umich.edu; liaos1@jxau.edu.cn

\section{Supporting Information}


6a, White solid. ${ }^{1} \mathrm{H}$ NMR (DMSO- $\left.d_{6}, 400 \mathrm{MHz}\right) \delta$ (ppm): 7.37 7.29 (1m, 8H, Ar-H), 3.56 (1d, 4H, $\left.J=16.0 \mathrm{~Hz}, 1-, 8-\mathrm{NHCH}_{2}\right), 1.71\left(1 \mathrm{~d}, 2 \mathrm{H}, J=16.0 \mathrm{~Hz}, 2-, 6-\mathrm{H}_{\mathrm{e}}\right), 1.51 \sim 1.34$ $\left(1 \mathrm{~m}, 6 \mathrm{H}, 3-, 5-\mathrm{H}_{\mathrm{e}}, 1-,{ }^{-\mathrm{NH}}, 2-, 6-\mathrm{H}_{\mathrm{a}}\right), 1.25(1 \mathrm{~m}, 1 \mathrm{H}, 4-\mathrm{H}), 1.15,1.14(2 \mathrm{t}, 2 \mathrm{H}, J=12.0 \mathrm{~Hz}$, 3-, 5- $\left.\mathrm{H}_{\mathrm{a}}\right), 1.01,0.97\left(2 \mathrm{~s}, 9 \mathrm{H}, 7-, 9-\mathrm{H}, 10-\mathrm{CH}_{3}\right) .{ }^{13} \mathrm{C}$ NMR (DMSO- $\left.d_{6}, 101 \mathrm{MHz}\right) \delta(\mathrm{ppm})$ : $141.80,141.74,130.84,130.81,129.80,128.03$ (Ar-C), 54.45 (8-C), 50.80 (1-C), 45.24 (4C), 44.76, 44.54 (1-, 8- $\mathrm{NHCH}_{2}$ ), 37.19 (2-C, 6-C), 28.40, 24.63 (7-, 9-, 10-C), 21.89 (3-C, 5-C). IR $\left(\mathrm{cm}^{-1}\right): 3045\left(\mathrm{w}, v_{=\mathrm{CH}}\right), 2948(\mathrm{~m}), 2926(\mathrm{~m}), 2858\left(\mathrm{~m}, v_{\mathrm{CH}}\right), 1599\left(\mathrm{~m}, \delta_{\mathrm{N}-\mathrm{H}}\right), 1504$ $\left(\mathrm{s}, v_{\mathrm{C}=\mathrm{C} \text { of aromatic ring }}\right), 1441\left(\mathrm{~m}, \tau_{\mathrm{CH} 2}\right), 1365\left(\mathrm{~m}, \tau_{\mathrm{CH} 3}\right), 1214(\mathrm{~s}), 1153\left(\mathrm{~m}, v_{\mathrm{C}-\mathrm{N}}\right), 824(\mathrm{~s}), 718$ (m); HRMS (ESI) for $\mathrm{C}_{24} \mathrm{H}_{33} \mathrm{~F}_{2} \mathrm{~N}_{2}$, calcd. 387.2612, found $387.2597[\mathrm{M}+\mathrm{H}]^{+}, \Delta=2.51 \mathrm{ppm}$.

6b, White solid. ${ }^{1} \mathrm{H}$ NMR (DMSO- $\left.d_{6}, 400 \mathrm{MHz}\right) \delta$ (ppm): 7.37 7.29 (1m, 8H, Ar-H), 3.57, $3.53\left(2 \mathrm{~d}, 4 \mathrm{H}, J=8.0 \mathrm{~Hz}, 1-, 8-\mathrm{NHCH}_{2}\right), 1.70\left(1 \mathrm{~d}, 2 \mathrm{H}, J=12.0 \mathrm{~Hz}, 2-, 6-\mathrm{H}_{\mathrm{e}}\right), 1.51(1 \mathrm{t}$, $1 \mathrm{H}, J=8.0 \mathrm{~Hz}, 1-$ or $8-\mathrm{NH}), 1.43 \sim 1.34\left(1 \mathrm{~m}, 5 \mathrm{H}, 3-, 5-\mathrm{H}_{\mathrm{e}}, 8-\right.$ or $1-\mathrm{NH}$ and 2-, $\left.6-\mathrm{H}_{\mathrm{a}}\right), 1.27$ (1m, 1H, 4-H), 1.14, $1.13\left(2 \mathrm{t}, 2 \mathrm{H}, J=14.0 \mathrm{~Hz}, 3-, 5-\mathrm{H}_{\mathrm{a}}\right), 1.00,0.97$ (2s, 9H, 7-, 9-H, 10$\left.\mathrm{CH}_{3}\right) .{ }^{13} \mathrm{C}$ NMR (DMSO-d $\left.6,101 \mathrm{MHz}\right) \delta(\mathrm{ppm}): 141.84,141.79,130.84,130.82,129.83$, 128.06 (Ar-C), 54.46 (8-C), 50.82 (1-C), 45.24 (4-C), 44.77, 44.55 (1-, 8- $\mathrm{NHCH}_{2}$ ), 37.20 (2-, 6-C), 28.42, 24.66 (7-, 9-, 10-C), $21.90\left(3-\right.$, 5-C). IR $\left(\mathrm{cm}^{-1}\right): 3045$ (w, v=CH), 2951 (m), $2923(\mathrm{~m}), 2843\left(\mathrm{~m}, v_{\mathrm{CH}}\right), 1599\left(\mathrm{w}, \delta_{\mathrm{N}-\mathrm{H}}\right), 1488\left(\mathrm{~s}, v_{\mathrm{C}=\mathrm{C} \text { of aromatic ring }}\right), 1430\left(\mathrm{~m}, \tau_{\mathrm{CH} 2}\right), 1365$ (m, $\left.\tau_{\mathrm{CH} 3}\right), 1186\left(\mathrm{~m}, v_{\mathrm{C}-\mathrm{N}}\right), 1088(\mathrm{~s}), 804(\mathrm{~s}), 729(\mathrm{~m})$; HRMS (ESI) for $\mathrm{C}_{24} \mathrm{H}_{33} \mathrm{Cl}_{2} \mathrm{~N}_{2}$, calcd. 419.2021, found $419.2023[\mathrm{M}+\mathrm{H}]^{+}, \Delta=-1.66 \mathrm{ppm}$.

6c, White solid. ${ }^{1} \mathrm{H}$ NMR (DMSO- $\left.d_{6}, 400 \mathrm{MHz}\right) \delta$ (ppm): 7.45 7.42, 7.31 7.27 (2m, 8H, Ar-H), 3.55, $3.52\left(2 \mathrm{~d}, 4 \mathrm{H}, J=8.0 \mathrm{~Hz}, 1-, 8-\mathrm{NHCH}_{2}\right), 1.70(1 \mathrm{~d}, 2 \mathrm{H}, J=12.0 \mathrm{~Hz}, 2-, 6-$ $\left.\mathrm{H}_{\mathrm{e}}\right), 1.52(1 \mathrm{t}, 1 \mathrm{H}, J=8.0 \mathrm{~Hz}$ and $1-$ or $8-\mathrm{NH}), 1.44 \sim 1.33\left(1 \mathrm{~m}, 5 \mathrm{H}, 3-, 5-\mathrm{H}_{\mathrm{e}}, 8-\right.$ or $1-\mathrm{NH}$ and 2-, 6- $\left.\mathrm{H}_{\mathrm{a}}\right), 1.25(1 \mathrm{~m}, 1 \mathrm{H}, 4-\mathrm{H}), 1.14,1.13\left(2 \mathrm{t}, 2 \mathrm{H}, J=12.0 \mathrm{~Hz}, 3-, 5-\mathrm{H}_{\mathrm{a}}\right), 1.00,0.96(2 \mathrm{~s}, 9 \mathrm{H}$, 


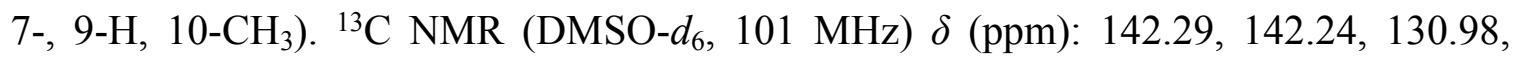
130.26, 130.25, 119.29, 119.26 (Ar-C), 54.47 (8-C), 50.83 (1-C), 45.26 (4-C), 44.82, 44.60 (1-, 8- $\left.\mathrm{NHCH}_{2}\right), 37.20$ (2-, 6-C), 28.43, 24.67 (7-, 9-, 10-C), 21.90 (3-, 5-C). IR ( $\left.\mathrm{cm}^{-1}\right)$ : $3059\left(\mathrm{w}, v_{=\mathrm{CH}}\right), 2954(\mathrm{~m}), 2915(\mathrm{~m}), 2836\left(\mathrm{~m}, v_{\mathrm{CH}}\right), 1588\left(\mathrm{w}, \delta_{\mathrm{N}-\mathrm{H}}\right), 1483\left(\mathrm{~s}, v_{\mathrm{C}=\mathrm{C} \text { of aromatic }}\right.$ ring $), 1434\left(\mathrm{~m}, \tau_{\mathrm{CH} 2}\right), 1365\left(\mathrm{~m}, \tau_{\mathrm{CH} 3}\right), 1186\left(\mathrm{~m}, v_{\mathrm{C}-\mathrm{N}}\right), 1067(\mathrm{~s}), 1006(\mathrm{~s}), 801(\mathrm{~s}), 718(\mathrm{~m})$; HRMS (ESI) for $\mathrm{C}_{24} \mathrm{H}_{33} \mathrm{Br}_{2} \mathrm{~N}_{2}$, calcd. 507.1010, found $507.0996[\mathrm{M}+\mathrm{H}]^{+}, \Delta=1.36 \mathrm{ppm}$.

6d, White solid. ${ }^{1} \mathrm{H}$ NMR (DMSO- $\left.d_{6}, 400 \mathrm{MHz}\right) \delta$ (ppm): 7.23 7.19, 7.09 7.06 (2m, 8H, Ar-H), 3.55, $3.51\left(2 \mathrm{~d}, 4 \mathrm{H}, J=8.0 \mathrm{~Hz}, 1-, 8-\mathrm{NHCH}_{2}\right), 2.26\left(1 \mathrm{~s}, 6 \mathrm{H}, 1-, 8-\mathrm{Ar}-\mathrm{CH}_{3}\right), 1.73$ (1d, $\left.2 \mathrm{H}, J=12.0 \mathrm{~Hz}, 2-, 6-\mathrm{H}_{\mathrm{e}}\right), 1.47 \sim 1.36\left(1 \mathrm{~m}, 4 \mathrm{H}, 3-, 5-\mathrm{H}_{\mathrm{e}}, 1-, 8-\mathrm{NH}\right), 1.29(1 \mathrm{t}, 2 \mathrm{H}, J=12.0$ $\left.\mathrm{Hz}, 2-, 6-\mathrm{H}_{\mathrm{a}}\right), 1.17,1.16\left(2 \mathrm{~m}, 3 \mathrm{H}, 3-, 5-\mathrm{H}_{\mathrm{a}}, 4-\mathrm{H}\right), 1.03,0.99\left(2 \mathrm{~s}, 9 \mathrm{H}, 7-, 9-\mathrm{H}, 10-\mathrm{CH}_{3}\right) .{ }^{13} \mathrm{C}$ NMR (DMSO- $\left.d_{6}, 101 \mathrm{MHz}\right) \delta(\mathrm{ppm}): 139.46,139.38,135.35,135.31,128.75,128.05$, 128.02 (Ar-C), 54.36 (8-C), 50.70 (1-C), 45.28 (4-C), 45.12, 45.04 (1-, 8- $\mathrm{NHCH}_{2}$ ), 37.19 (2-, 6-C), 28.37, 24.67 (7-, 9-, 10-C), $21.86\left(3-\right.$, 5-C), $20.87\left(\mathrm{Ar}^{-} \mathrm{CH}_{3}\right) . \mathrm{IR}\left(\mathrm{cm}^{-1}\right): 3048$ (w, $\left.v_{=\mathrm{CH}}\right), 2963(\mathrm{~m}), 2923(\mathrm{~m}), 2858\left(\mathrm{~m}, v_{\mathrm{CH}}\right), 1642\left(\mathrm{~m}, \delta_{\mathrm{N}-\mathrm{H}}\right), 1513\left(\mathrm{~s}, v_{\mathrm{C}=\mathrm{C} \text { of aromatic ring }}\right), 1437$ (m, $\left.\tau_{\mathrm{CH} 2}\right), 1376\left(\mathrm{~m}, \tau_{\mathrm{CH} 3}\right), 1189\left(\mathrm{~m}, v_{\mathrm{C}-\mathrm{N}}\right), 1092(\mathrm{~m}), 797(\mathrm{~s}), 725(\mathrm{~m})$; HRMS (ESI) for $\mathrm{C}_{26} \mathrm{H}_{39} \mathrm{~N}_{2}$, calcd. 379.3113 , found $379.3114[\mathrm{M}+\mathrm{H}]^{+}, \Delta=-1.25 \mathrm{ppm}$.

6e. ${ }^{1} \mathrm{H}$ NMR (DMSO- $\left.d_{6}, 400 \mathrm{MHz}\right) \delta(\mathrm{ppm}):$ 7.23 7.18, 6.82 6.79 (2m, $8 \mathrm{H}$, Ar-H), $3.68\left(2 \mathrm{~s}, 6 \mathrm{H}, 1-, 8-\mathrm{ArOCH}_{3}\right), 3.48\left(1 \mathrm{~d}, 4 \mathrm{H}, J=16.0 \mathrm{~Hz}, 1-, 8-\mathrm{NHCH}_{2}\right), 1.69$ (1d, 2H, $J=12.0$ $\left.\mathrm{Hz}, 2-, 6-\mathrm{H}_{\mathrm{e}}\right), 1.44 \sim 1.33\left(1 \mathrm{~m}, 4 \mathrm{H}, 3-, 5-\mathrm{H}_{\mathrm{e}}, 1-, 8-\mathrm{NH}\right), 1.28 \sim 1.19\left(1 \mathrm{~m}, 2 \mathrm{H}, 2-, 6-\mathrm{H}_{\mathrm{a}}\right), 1.13$, $1.12\left(2 \mathrm{~m}, 3 \mathrm{H}, 3-, 5-\mathrm{H}_{\mathrm{a}}, 4-\mathrm{H}\right), 1.00,0.96\left(2 \mathrm{~s}, 9 \mathrm{H}, 7-, 9-\mathrm{H}, 10-\mathrm{CH}_{3}\right) .{ }^{13} \mathrm{C}$ NMR (DMSO-d 6 , $101 \mathrm{MHz}) \delta(\mathrm{ppm}): 158.06,158.04,134.39,134.34,129.17,129.16,113.59,113.58$ (ArC), 55.15 (8-C), 54.31 (1-, 8- $\left.\mathrm{OCH}_{3}\right), 50.67$ (1-C), 45.10, 44.96 (1-, 8- $\left.\mathrm{NHCH}_{2}\right), 44.72$ (4C), 37.20 (2-, 6-C), 28.37, 24.69 (7-, 9-, 10-C), 21.88 (3-, 5-C). IR ( $\left.\mathrm{cm}^{-1}\right): 3029$ (w, $v_{=\mathrm{CH}}$ ), 
$2959(\mathrm{~m}), 2932(\mathrm{~m}), 2835\left(\mathrm{~m}, v_{\mathrm{CH}}\right), 1610\left(\mathrm{~m}, \delta_{\mathrm{N}-\mathrm{H}}\right), 1511\left(\mathrm{~m}, v_{\mathrm{c}=\mathrm{c} \text { of aromatic ring }}\right), 1438(\mathrm{~m}$, $\left.\tau_{\mathrm{CH} 2}\right), 1379\left(\mathrm{~m}, \tau_{\mathrm{CH} 3}\right), 1244(\mathrm{~s}), 1179\left(\mathrm{~m}, v_{\mathrm{C}-\mathrm{N}}\right), 1031(\mathrm{~s}), 816(\mathrm{~s}), 726(\mathrm{~m})$; HRMS (ESI) for $\mathrm{C}_{26} \mathrm{H}_{39} \mathrm{~N}_{2} \mathrm{O}_{2}$, calcd. 411.3011, found $411.3014[\mathrm{M}+\mathrm{H}]^{+}, \Delta=-2.03$ ppm.

6f, White solid. ${ }^{1} \mathrm{H}$ NMR (DMSO- $\left.d_{6}, 400 \mathrm{MHz}\right) \delta$ (ppm): $7.63 \sim 7.55$ (2m, 8H, Ar-H), $3.69\left(1 \mathrm{~d}, 4 \mathrm{H}, J=16.0 \mathrm{~Hz}, 1-, 8-\mathrm{NHCH}_{2}\right), 1.75\left(1 \mathrm{~d}, 2 \mathrm{H}, J=12.0 \mathrm{~Hz}, 2-, 6-\mathrm{H}_{\mathrm{e}}\right), 1.60 \sim 1.41$ $\left(1 \mathrm{~m}, 6 \mathrm{H}, 3-, 5-\mathrm{H}_{\mathrm{e}}, 1-,, 8-\mathrm{NH}, 2-, 6-\mathrm{H}_{\mathrm{a}}\right), 1.32 \sim 1.28(1 \mathrm{~m}, 1 \mathrm{H}, 4-\mathrm{H}), 1.22 \sim 1.14(1 \mathrm{~m}, 2 \mathrm{H}, 3-$, 5- $\left.\mathrm{H}_{\mathrm{a}}\right), 1.04,1.02\left(2 \mathrm{~s}, 9 \mathrm{H}, 7-, 9-\mathrm{H}, 10-\mathrm{CH}_{3}\right) .{ }^{13} \mathrm{C}$ NMR (DMSO- $\left.d_{6}, 101 \mathrm{MHz}\right) \delta(\mathrm{ppm})$ : 148.52, 129.35, 129.33, 125.63, 125.59 (Ar-C), $125.56\left(\mathrm{Ar}_{-} \mathrm{CF}_{3}\right), 55.21$ (8-C), 51.55 (1-C), 46.18 (4-C), 45.88, 45.59 (1-, 8- $\left.\mathrm{NHCH}_{2}\right), 37.92$ (2-, 6-C), 29.06, 25.33 (7-, 9-, 10-C), 22.62 (3-, 5-C). IR ( $\left.\mathrm{cm}^{-1}\right): 3059\left(\mathrm{w}, v_{=\mathrm{CH}}\right), 2966(\mathrm{~m}), 2929(\mathrm{~m}), 2865\left(\mathrm{~m}, v_{\mathrm{CH}}\right), 1617\left(\mathrm{~m}, \delta_{\mathrm{N}-\mathrm{H}}\right)$, $1462\left(\mathrm{~m}, \tau_{\mathrm{CH} 2}\right.$ and $\left.v_{\mathrm{C}=\mathrm{C} \text { of aromatic ring }}\right), 1322\left(\mathrm{~s}, \tau_{\mathrm{CH} 3}\right), 1153\left(\mathrm{~m}, v_{\mathrm{C}-\mathrm{N}}\right), 1110(\mathrm{~s}), 821(\mathrm{~m}), 729$ (m); HRMS (ESI) for $\mathrm{C}_{26} \mathrm{H}_{33} \mathrm{~F}_{6} \mathrm{~N}_{2}$, calcd. 487.2548, found $487.2555[\mathrm{M}+\mathrm{H}]^{+}, \Delta=-2.55$ ppm.

6g, Light yellow solid. ${ }^{1} \mathrm{H}$ NMR (DMSO- $\left.d_{6}, 400 \mathrm{MHz}\right) \delta$ (ppm): 7.14 7.09, 6.65 6.62 (2m, 8H, Ar-H), 3.46, $3.43\left(2 \mathrm{~d}, 4 \mathrm{H}, J=8.0 \mathrm{~Hz}, 1-, 8-\mathrm{NHCH}_{2}\right), 2.82\left(1 \mathrm{~s}, 12 \mathrm{H}, 1-, 8-\mathrm{N}\left(\mathrm{CH}_{3}\right)_{2}\right)$, $1.71\left(1 \mathrm{~d}, 2 \mathrm{H}, J=12.0 \mathrm{~Hz}, 2-, 6-\mathrm{H}_{\mathrm{e}}\right), 1.46 \sim 1.38\left(1 \mathrm{~m}, 6 \mathrm{H}, 3-, 5-\mathrm{H}_{\mathrm{e}}, 1-, 8-\mathrm{NH}, 2-, 6-\mathrm{H}_{\mathrm{a}}\right)$, 1.18 1.11 (1m, 3H, 3-, 5- $\left.\mathrm{H}_{\mathrm{a}}, 4-\mathrm{H}\right), 1.03,0.98(2 \mathrm{~s}, 9 \mathrm{H}, 7-, \text { 9-H, 10-CH })^{13}{ }^{13} \mathrm{C}$ NMR (DMSO$\left.d_{6}, 101 \mathrm{MHz}\right) \delta(\mathrm{ppm}): 149.56,130.02,128.87,128.82,112.59$ (Ar-C), 54.25 (8-C), 50.62 (1-C), 45.07, 44.83 (1-, 8- $\left.\mathrm{NHCH}_{2}, 4-\mathrm{C}\right), 40.62$ (1-, 8-N( $\left.\left(\mathrm{CH}_{3}\right)_{2}\right), 37.20$ (2-, 6-C), 28.38, 24.75 (7-, 9-, 10-C), 21.86 (3-, 5-C). IR ( $\left.\mathrm{cm}^{-1}\right): 3076$ (w, $\left.v_{=\mathrm{CH}}\right), 2948$ (m), 2925 (m), 2852 $\left(\mathrm{m}, v_{\mathrm{CH}}\right), 1613\left(\mathrm{~s}, \delta_{\mathrm{N}-\mathrm{H}}\right), 1522\left(\mathrm{~s}, v_{\mathrm{C}=\mathrm{C} \text { of aromatic ring }}\right), 1437\left(\mathrm{~m}, \tau_{\mathrm{CH} 2}\right), 1347\left(\mathrm{~s}, \tau_{\mathrm{CH} 3}\right), 1166(\mathrm{~m}$, $v_{\text {C-N }}$ ), $801(\mathrm{~m}), 722(\mathrm{~m})$; HRMS (ESI) for $\mathrm{C}_{28} \mathrm{H}_{45} \mathrm{~N}_{4}$, calcd. 437.3644, found 437.3658 $[\mathrm{M}+\mathrm{H}]^{+}, \Delta=-4.41 \mathrm{ppm}$. 
6h, White solid. ${ }^{1} \mathrm{H}$ NMR (DMSO- $\left.d_{6}, 400 \mathrm{MHz}\right) \delta$ (ppm): 7.31 7.27, 7.20 7.17 (2m, 8H, Ar-H), 3.56, 3.53 (2d, 4H, $\left.J=16.0 \mathrm{~Hz}, 1-, 8-\mathrm{NHCH}_{2}\right), 2.45$ (1s, 6H, 1-, 8- $\left.\mathrm{SCH}_{3}\right), 1.73$ (1d, $\left.2 \mathrm{H}, J=12.0 \mathrm{~Hz}, 2-, 6-\mathrm{H}_{\mathrm{e}}\right), 1.47 \sim 1.37\left(1 \mathrm{~m}, 5 \mathrm{H}, 3-, 5-\mathrm{H}_{\mathrm{e}}, 1-\right.$ or $8-\mathrm{NH}$ and 2-, 6- $\mathrm{H}_{\mathrm{a}}$ ), $1.31 \sim 1.28(1 \mathrm{~m}, 2 \mathrm{H}, 8-$ or $1-\mathrm{NH}$ and $4-\mathrm{H}), 1.20 \sim 1.13\left(1 \mathrm{~m}, 2 \mathrm{H}, 3-, 5-\mathrm{H}_{\mathrm{a}}\right), 1.03,1.00(2 \mathrm{~s}, 9 \mathrm{H}$, 7-, 9-H, 10- $\mathrm{CH}_{3}$ ). ${ }^{13} \mathrm{C}$ NMR (DMSO- $\left.d_{6}, 101 \mathrm{MHz}\right) \delta(\mathrm{ppm}): 139.53,135.67,135.60,128.77$, 128.73, 126.25, 126.22 (Ar-C), 54.40 (8-C), 50.75 (1-C), 45.23, 45.07 (1-, 8- $\mathrm{NHCH}_{2}$ ), 44.84 (4-C), 37.20 (2-, 6-C), 28.40, 24.70 (7-, 9-, 10-C), 21.88 (3-, 5-C), 15.35, 15.30 (1-, 8- $\left.\mathrm{SCH}_{3}\right) . \mathrm{IR}\left(\mathrm{cm}^{-1}\right): 3070$ (w, $\left.v_{=\mathrm{CH}}\right), 2954(\mathrm{~m}), 2920(\mathrm{~m}), 2837$ (m, $\left.v_{\mathrm{CH}}\right), 1645\left(\mathrm{~m}, \delta_{\mathrm{N}-\mathrm{H}}\right)$, $1599\left(\mathrm{~m}, v_{\mathrm{C}=\mathrm{C} \text { of aromatic ring }}\right), 1434\left(\mathrm{~m}, \tau_{\mathrm{CH} 2}\right), 1375\left(\mathrm{~m}, \tau_{\mathrm{CH} 3}\right), 1111\left(\mathrm{~m}, v_{\mathrm{C}-\mathrm{N}}\right), 887(\mathrm{~s}), 731(\mathrm{~s})$; HRMS (ESI) for $\mathrm{C}_{26} \mathrm{H}_{39} \mathrm{~N}_{2} \mathrm{~S}_{2}$, calcd. 443.2554, found $443.2555[\mathrm{M}+\mathrm{H}]^{+}, \Delta=-1.25 \mathrm{ppm}$.

6i, White solid. ${ }^{1} \mathrm{H}$ NMR (DMSO- $\left.d_{6}, 400 \mathrm{MHz}\right) \delta$ (ppm): 7.85 7.82, 7.65 7.57, 7.42 7.39 (3m, 8H, Ar-H), 3.75 (2d, 4H, J=20.0 Hz, 1-, 8- $\mathrm{NHCH}_{2}$ ), 1.73 (1d, 2H, $J=12.0$ $\left.\mathrm{Hz}, 2-, 6-\mathrm{H}_{\mathrm{e}}\right), 1.50 \sim 1.37\left(1 \mathrm{~m}, 6 \mathrm{H}, 3-, 5-\mathrm{H}_{\mathrm{e}}, 1-, 8-\mathrm{NH}, 2-, 6-\mathrm{H}_{\mathrm{a}}\right), 1.31 \sim 1.28(1 \mathrm{~m}, 1 \mathrm{H}, 4-\mathrm{H})$, 1.22 1.15 (1m, 2H, 3-, 5- $\left.\mathrm{H}_{\mathrm{a}}\right), 1.04,1.00\left(2 \mathrm{~s}, 9 \mathrm{H}, 7-, 9-\mathrm{H}, 10-\mathrm{CH}_{3}\right) .{ }^{13} \mathrm{C}$ NMR (DMSO-d 6 , $101 \mathrm{MHz}) \delta$ (ppm): 141.86, 141.79, 133.10, 131.78, 131.55, 127.63, 127.56, 126.93, $126.15,126.09,124.21$ (Ar-C), 126.11, $126.05\left(\mathrm{Ar}_{-} \mathrm{CF}_{3}\right), 55.38$ (8-C), 51.74 (1-, 8$\mathrm{NHCH}_{2}$ ), 46.12 (1-C), 42.12, 42.07 (2-, 6-C), 37.85 (4-C), 28.88, 25.08 (7-, 9-, 10-C), 22.52 (3-, 5-C). IR ( $\left.\mathrm{cm}^{-1}\right): 3069$ (w, $v_{=\mathrm{CH}}$ ), 2955 (m), 2929 (m), 2865 (m, $\left.v_{\mathrm{CH}}\right), 1609$ (s, $\left.\delta_{\mathrm{N}-\mathrm{H}}\right), 1455\left(\mathrm{~m}, \tau_{\mathrm{CH} 2}\right.$ and $\left.v_{\mathrm{C}=\mathrm{C} \text { of aromatic ring }}\right), 1311\left(\mathrm{~s}, \tau_{\mathrm{CH} 3}\right), 1154\left(\mathrm{~m}, v_{\mathrm{C}-\mathrm{N}}\right), 1109(\mathrm{~s}), 1036(\mathrm{~s})$, 765 (s), 696 (m); HRMS (ESI) for $\mathrm{C}_{26} \mathrm{H}_{33} \mathrm{~F}_{6} \mathrm{~N}_{2}$, calcd. 487.2548, found $487.2559[\mathrm{M}+\mathrm{H}]^{+}$, $\Delta=-3.40 \mathrm{ppm}$.

6j, Light yellow viscous oil. ${ }^{1} \mathrm{H}$ NMR (DMSO- $\left.d_{6}, 400 \mathrm{MHz}\right) \delta(\mathrm{ppm}): 7.51 \sim 7.50$, 6.36 6.34, 6.21 6.19 (3m, 6H, furan-H), $3.60\left(2 \mathrm{~d}, 4 \mathrm{H}, J=8.0 \mathrm{~Hz}, 1-, 8-\mathrm{NHCH}_{2}\right), 1.70(1 \mathrm{~d}$, 
$\left.2 \mathrm{H}, J=16.0 \mathrm{~Hz}, 2-, 6-\mathrm{H}_{\mathrm{e}}\right), 1.45 \sim 1.24$ (3m, 7H, 3-, 5- $\mathrm{H}_{\mathrm{e}}, 1-$, , 8-NH, 2-, 6- $\left.\mathrm{H}_{\mathrm{a}}, 4-\mathrm{H}\right), 1.19 \sim 1.12$ $\left(1 \mathrm{~m}, 2 \mathrm{H}, 3-, 5-\mathrm{H}_{\mathrm{a}}\right), 1.01,0.98\left(2 \mathrm{~s}, 9 \mathrm{H}, 7-, 9-\mathrm{H}, 10-\mathrm{CH}_{3}\right) .{ }^{13} \mathrm{C}$ NMR (DMSO-d, $\left.101 \mathrm{MHz}\right)$ $\delta(\mathrm{ppm}): 155.85,155.67,141.43,110.35,110.31,105.81,105.80$ (furan-C), 54.20 (8-C), 50.59 (1-C), 44.95 (4-C), 38.75, 38.61 (1-, 8- $\left.\mathrm{NHCH}_{2}\right), 37.08$ (2-, 6-C), 28.03, 24.33 (7-, 9-, 10-C), $21.78\left(3-\right.$, 5-C). IR $\left(\mathrm{cm}^{-1}\right): 3134\left(\mathrm{w}, v_{=\mathrm{CH}}\right), 2945(\mathrm{~m}), 2929(\mathrm{~m}), 2833\left(\mathrm{~m}, v_{\mathrm{CH}}\right)$, $1598\left(\mathrm{~m}, \delta_{\mathrm{N}-\mathrm{H}}\right), 1507\left(\mathrm{~m}, v_{\mathrm{C}=\mathrm{C} \text { of aromatic ring }}\right), 1447\left(\mathrm{~m}, \tau_{\mathrm{CH} 2}\right), 1341\left(\mathrm{~m}, \tau_{\mathrm{CH} 3}\right), 1139\left(\mathrm{~m}, v_{\mathrm{C}-\mathrm{N}}\right)$, 1103 (s, $v_{\mathrm{C}-\mathrm{O}}$ ), 992 (s), 801 (s), 741 (s); HRMS (ESI) for $\mathrm{C}_{20} \mathrm{H}_{31} \mathrm{~N}_{2} \mathrm{O}_{2}$, calcd. 331.2385, found $331.2397[\mathrm{M}+\mathrm{H}]^{+}, \Delta=-5.08 \mathrm{ppm}$.

6k, Light yellow viscous oil. ${ }^{1} \mathrm{H}$ NMR (DMSO- $\left.d_{6}, 400 \mathrm{MHz}\right) \delta(\mathrm{ppm}): 6.42 \sim 6.41$, 6.26 6.25 (2m, 4H, furan-H), $3.57\left(2 \mathrm{~d}, 4 \mathrm{H}, J=12.0 \mathrm{~Hz}, 1-, 8-\mathrm{NHCH}_{2}\right), 1.67(1 \mathrm{~d}, 2 \mathrm{H}, J=12.0$ $\left.\mathrm{Hz}, 2-, 6-\mathrm{H}_{\mathrm{e}}\right), 1.48 \sim 1.20\left(3 \mathrm{~m}, 7 \mathrm{H}, 3-, 5-\mathrm{H}_{\mathrm{e}}, 1-\right.$, 8-NH, 2-, 6-H 3-, 5- $\left.\mathrm{H}_{\mathrm{a}}\right), 0.98,0.96\left(2 \mathrm{~s}, 9 \mathrm{H}, 7-, 9-\mathrm{H}, 10-\mathrm{CH}_{3}\right) .{ }^{13} \mathrm{C}$ NMR (DMSO- $\left.d_{6}, 101 \mathrm{MHz}\right) \delta(\mathrm{ppm})$ : $158.50,158.32,119.05,119.04,112.13,108.95,108.86$ (furan-C), 54.33 (8-C), 50.67 (1C), 44.86 (4-C), 38.76, 38.61 (1-, 8- $\mathrm{NHCH}_{2}$ ), 37.02 (2-, 6-C), 28.04, 24.29 (7-, 9-, 10-C), $21.72\left(3-\right.$, 5-C). IR $\left(\mathrm{cm}^{-1}\right): 3134\left(\mathrm{w}, v_{=\mathrm{CH}}\right), 2959(\mathrm{~m}), 2929(\mathrm{~m}), 2858\left(\mathrm{~m}, v_{\mathrm{CH}}\right), 1599(\mathrm{~s}$, $\left.\delta_{\mathrm{N}-\mathrm{H}}\right), 1507\left(\mathrm{~s}, v_{\mathrm{C}=\mathrm{C} \text { of aromatic ring }}\right), 1458(\mathrm{~m}), 1442\left(\mathrm{~m}, \tau_{\mathrm{CH} 2}\right), 1382\left(\mathrm{~m}, \tau_{\mathrm{CH} 3}\right), 1193(\mathrm{~m}), 1178$ (m, $v_{\mathrm{C}-\mathrm{N}}$ ), $1123(\mathrm{~s}), 778$ (s), 715 (m); HRMS (ESI) for $\mathrm{C}_{20} \mathrm{H}_{29} \mathrm{Br}_{2} \mathrm{~N}_{2} \mathrm{O}_{2}$, calcd. 487.0596, found $487.0605[\mathrm{M}+\mathrm{H}]^{+}, \Delta=-3.56 \mathrm{ppm}$.

61, Light yellow viscous oil. ${ }^{1} \mathrm{H}$ NMR (DMSO- $\left.d_{6}, 400 \mathrm{MHz}\right) \delta(\mathrm{ppm})$ : 6.03 6.01, 5.91 5.90 (2m, 4H, furan-H), $3.53\left(2 \mathrm{~d}, 4 \mathrm{H}, J=12.0 \mathrm{~Hz}, 1-, 8-\mathrm{NHCH}_{2}\right), 2.21,2.20(2 \mathrm{~s}, 6 \mathrm{H}$, furan- $\left.\mathrm{CH}_{3}\right), 1.68\left(1 \mathrm{~d}, 2 \mathrm{H}, J=12.0 \mathrm{~Hz}, 2-, 6-\mathrm{H}_{\mathrm{e}}\right), 1.43 \sim 1.18\left(3 \mathrm{~m}, 7 \mathrm{H}, 3-, 5-\mathrm{H}_{\mathrm{e}}, 1-, 8-\mathrm{NH}, 2-\right.$, 6- $\left.\mathrm{H}_{\mathrm{a}}, 4-\mathrm{H}\right), 1.16 \sim 1.09\left(1 \mathrm{~m}, 2 \mathrm{H}, 3-, 5-\mathrm{H}_{\mathrm{a}}\right), 0.99,0.96\left(2 \mathrm{~s}, 9 \mathrm{H}, 7-, 9-\mathrm{H}, 10-\mathrm{CH}_{3}\right) .{ }^{13} \mathrm{C} \mathrm{NMR}$ $\left(\mathrm{DMSO}-d_{6}, 101 \mathrm{MHz}\right) \delta(\mathrm{ppm}): 153.94,153.78,149.95,149.90,106.46,106.40,106.17$, 
106.15 (furan-C), 54.16 (8-C), 50.53 (1-C), 44.89 (4-C), 38.80, 38.63 (1-, 8- $\mathrm{NHCH}_{2}$ ), 37.06 (2-, 6-C), 27.99, 24.29 (7-, 9-, 10-C), 21.75 (3-, 5-C), 13.37, 13.34 (furan- $\left.\mathrm{CH}_{3}\right)$. IR $\left(\mathrm{cm}^{-1}\right): 3102\left(\mathrm{w}, v_{=\mathrm{CH}}\right), 2952(\mathrm{~m}), 2921(\mathrm{~m}), 2859\left(\mathrm{~m}, v_{\mathrm{CH}}\right), 1617\left(\mathrm{~m}, \delta_{\mathrm{N}-\mathrm{H}}\right), 1573\left(\mathrm{~m}, v_{\mathrm{C}=\mathrm{C}}\right.$ of aromatic ring $), 1441\left(\mathrm{~m}, \tau_{\mathrm{CH} 2}\right), 1383,1364\left(\mathrm{~m}, \tau_{\mathrm{CH} 3}\right), 1179\left(\mathrm{~m}, v_{\mathrm{C}-\mathrm{N}}\right), 1078\left(\mathrm{~m}, v_{\mathrm{C}-\mathrm{O}}\right), 1020$ (m), 777 (s), 700 (m); HRMS (ESI) for $\mathrm{C}_{22} \mathrm{H}_{35} \mathrm{~N}_{2} \mathrm{O}_{2}$, calcd. 359.2699, found 359.2690 $[\mathrm{M}+\mathrm{H}]^{+}, \Delta=0.75 \mathrm{ppm}$.

6m, Light green viscous oil. ${ }^{1} \mathrm{H}$ NMR (DMSO- $\left.d_{6}, 400 \mathrm{MHz}\right) \delta(\mathrm{ppm}): 8.55 \sim 8.52$, 8.42 8.40, 7.76 7.72, 7.32 7.29 (4m, 8H, pyridine-H), 3.62 (1d, 4H, J=16.0 Hz, 1-, 8$\left.\mathrm{NHCH}_{2}\right), 1.75 \sim 1.62\left(1 \mathrm{~m}, 4 \mathrm{H}, 1-\right.$, 8-NH, 2-, 6- $\left.\mathrm{H}_{\mathrm{e}}\right), 1.45 \sim 1.37$ (1m, 4H, 3-, 5- $\mathrm{H}_{\mathrm{e}}, 2-$, 6- $\mathrm{H}_{\mathrm{a}}$ ), 1.33 1.27 (1m, 1H, 4-H, 3-, 5- $\left.\mathrm{H}_{\mathrm{a}}\right), 1.23 \sim 1.14\left(1 \mathrm{~m}, 1 \mathrm{H}, 4-\mathrm{H}, 3-, 5-\mathrm{H}_{\mathrm{a}}\right), 1.00,1.05(2 \mathrm{~s}, 9 \mathrm{H}$, 7-, 9-H, 10- $\left.\mathrm{CH}_{3}\right) .{ }^{13} \mathrm{C}$ NMR (DMSO- $\left.d_{6}, 101 \mathrm{MHz}\right) \delta(\mathrm{ppm}): 149.57,149.52,147.78,147.75$, 138.02, 137.94, 135.78, 135.77, 123.39 (pyridine-C), 54.58 (8-C), 50.90 (1-C), 45.19 (4C), 43.02, $42.83\left(1-\right.$, 8- $\left.\mathrm{NHCH}_{2}\right), 37.22$ (2-, 6-C), 28.38, 24.60 (7-, 9-, 10-C), 21.93 (3-, 5C). IR ( $\left.\mathrm{cm}^{-1}\right): 3294\left(\mathrm{br}, v_{\mathrm{N}-\mathrm{H}}\right), 3029\left(\mathrm{~m}, v_{=\mathrm{CH}}\right), 2952(\mathrm{~m}), 2929(\mathrm{~m}), 2859\left(\mathrm{~m}, v_{\mathrm{CH}}\right), 1645$ $\left(\mathrm{w}, \delta_{\mathrm{N}-\mathrm{H}}\right), 1577\left(\mathrm{~m}, v_{\mathrm{C}=\mathrm{C} \text { of aromatic ring }}\right), 1426\left(\mathrm{~m}, \tau_{\mathrm{CH} 2}\right), 1364\left(\mathrm{~m}, \tau_{\mathrm{CH} 3}\right), 1183\left(\mathrm{~m}, v_{\mathrm{C}-\mathrm{N}}\right), 1028$ (m), 792 (m), 711 (s); HRMS (ESI) for $\mathrm{C}_{22} \mathrm{H}_{33} \mathrm{~N}_{4}$, calcd. 353.2705, found 353.2707 $[\mathrm{M}+\mathrm{H}]^{+}, \Delta=-2.03 \mathrm{ppm}$.

6n, White solid. ${ }^{1} \mathrm{H}$ NMR (DMSO- $\left.d_{6}, 400 \mathrm{MHz}\right) \delta$ (ppm): 8.46 8.44, 7.40 7.36 (2m, 8H, pyridine-H), $3.63\left(1 \mathrm{~d}, 4 \mathrm{H}, J=16.0 \mathrm{~Hz}, 1-, 8-\mathrm{NH}_{-} \mathrm{CH}_{2}\right), 1.74 \sim 1.71\left(1 \mathrm{~m}, 4 \mathrm{H}, 2-, 6-\mathrm{H}_{\mathrm{e}}\right.$, 1-, 8-NH), 1.46 1.38 (1m, 4H, 3-, 5- $\mathrm{H}_{\mathrm{e}}, 2-$, 6- $\left.\mathrm{H}_{\mathrm{a}}\right), 1.31 \sim 1.13\left(1 \mathrm{~m}, 3 \mathrm{H}, 4-\mathrm{H}, 3-, 5-\mathrm{H}_{\mathrm{a}}\right), 1.02$, 0.99 (2s, 9H, 7-, 9-H, 10-CH CH. $^{13} \mathrm{C}$ NMR (DMSO- $\left.d_{6}, 101 \mathrm{MHz}\right) \delta(\mathrm{ppm}): 152.06,152.01$, 149.36, 123.21, 123.15 (pyridine-C), 54.54 (8-C), 50.86 (1-C), 45.39 (4-C), 44.42, 44.22 (1-, 8- $\left.\mathrm{NHCH}_{2}\right), 37.20$ (2-, 6-C), 28.44, 24.59 (7-, 9-, 10-C), 21.93 (3-, 5-C). IR (cm-1): 
$3299\left(\mathrm{br}, v_{\mathrm{N}-\mathrm{H}}\right), 3029\left(\mathrm{~m}, v_{=\mathrm{CH}}\right), 2959(\mathrm{~m}), 2922(\mathrm{~m}), 2851\left(\mathrm{~m}, v_{\mathrm{CH}}\right), 1642\left(\mathrm{w}, \delta_{\mathrm{N}-\mathrm{H}}\right), 1596$

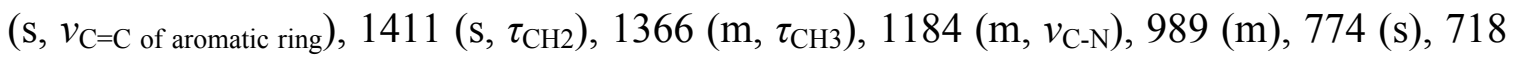
(s); HRMS (ESI) for $\mathrm{C}_{22} \mathrm{H}_{33} \mathrm{~N}_{4}$, calcd. 353.2705, found $353.2695[\mathrm{M}+\mathrm{H}]^{+}, \Delta=1.28 \mathrm{ppm}$.

6o, White solid. ${ }^{1} \mathrm{H} \mathrm{NMR}\left(\mathrm{CDCl}_{3}, 400 \mathrm{MHz}\right) \delta(\mathrm{ppm}): 7.30 \sim 7.29,6.92 \sim 6.91(2 \mathrm{~m}, 6 \mathrm{H}$, thiophene-H), $3.79\left(1 \mathrm{~d}, 4 \mathrm{H}, J=16.0 \mathrm{~Hz}, 1-, 8-\mathrm{NHCH}_{2}\right), 1.73 \sim 1.65\left(1 \mathrm{~m}, 4 \mathrm{H}, 2-, 6-\mathrm{H}_{\mathrm{e}}, 1-\right.$, - $^{-}$ $\mathrm{NH}), 1.46 \sim 1.44\left(1 \mathrm{~m}, 4 \mathrm{H}, 3-, 5-\mathrm{H}_{\mathrm{e}}, 2-, 6-\mathrm{H}_{\mathrm{a}}\right), 1.28 \sim 1.25,1.19 \sim 1.12(2 \mathrm{~m}, 3 \mathrm{H}, 4-\mathrm{H}, 3-, 5-$ $\left.\mathrm{H}_{\mathrm{a}}\right), 1.02,1.01\left(2 \mathrm{~s}, 9 \mathrm{H}, 7-, 9-\mathrm{H}, 10-\mathrm{CH}_{3}\right) \cdot{ }^{13} \mathrm{C}$ NMR (DMSO- $\left.d_{6}, 101 \mathrm{MHz}\right) \delta(\mathrm{ppm}): 147.78$, $147.43,126.58,126.57,124.19,124.07,123.38,123.21$ (thiophene-C), 54.58 (8-C), 50.83 (1-C), 45.14 (4-C), 40.76, 40.52 (1-, 8- $\mathrm{NHCH}_{2}$ ), 37.13 (2-, 6-C), 28.24, 24.51 (7-, 9-, 10C), 21.84 (3-, 5-C). IR ( $\left.\mathrm{cm}^{-1}\right): 3095\left(\mathrm{~m}, v_{=\mathrm{CH}}\right), 2944(\mathrm{~m}), 2930(\mathrm{~m}), 2865\left(\mathrm{~m}, v_{\mathrm{CH}}\right), 1599$ $\left(\mathrm{w}, \delta_{\mathrm{N}-\mathrm{H}}\right), 1479(\mathrm{w}), 1461\left(\mathrm{~m}, v_{\mathrm{C}=\mathrm{C} \text { of aromatic ring }}\right), 1441\left(\mathrm{~m}, \tau_{\mathrm{CH} 2}\right), 1364\left(\mathrm{~m}, \tau_{\mathrm{CH} 3}\right), 1180(\mathrm{~m}$, $v_{\text {C-N }}$ ), $818(\mathrm{~m}), 705$ (s); HRMS (ESI) for $\mathrm{C}_{20} \mathrm{H}_{31} \mathrm{~N}_{2} \mathrm{~S}_{4}$, calcd. 363.1929, found 363.1933 $[\mathrm{M}+\mathrm{H}]^{+}, \Delta=-2.69 \mathrm{ppm}$.

6p, Light yellow solid. ${ }^{1} \mathrm{H}$ NMR (DMSO- $\left.d_{6}, 400 \mathrm{MHz}\right) \delta(\mathrm{ppm}): 10.49(1 \mathrm{~s}, 2 \mathrm{H}$, pyrrole-NH), 6.59 6.56, 5.89 5.86, 5.82 5.80 (3m, 6H, pyrrole-H), $3.52(1 \mathrm{~d}, 4 \mathrm{H}, J=8.0$ $\left.\mathrm{Hz}, 1-, 8-\mathrm{NHCH}_{2}\right), 1.70\left(1 \mathrm{~d}, 2 \mathrm{H}, J=12.0 \mathrm{~Hz}, 2-, 6-\mathrm{H}_{\mathrm{e}}\right), 1.45\left(1 \mathrm{~d}, 2 \mathrm{H}, J=12.0 \mathrm{~Hz}, 3-, 5-\mathrm{H}_{\mathrm{e}}\right)$, 1.35 1.11 (2m, 7H, 1-, 8-NH, 2-, 6- $\mathrm{H}_{\mathrm{a}}, 4-\mathrm{H}, 3-$, 5- $\left.\mathrm{H}_{\mathrm{a}}\right), 1.01,0.97(2 \mathrm{~s}, 9 \mathrm{H}, 7-, \text { 9-H, 10-CH })_{3}$. ${ }^{13} \mathrm{C}$ NMR (DMSO- $\left.d_{6}, 101 \mathrm{MHz}\right) \delta(\mathrm{ppm}): 132.08,131.92,116.46,116.41,107.20,107.08$, 104.97 (pyrrole-C), 54.20 (8-C), 50.62 (1-C), 45.15 (4-C), 38.72, $38.58\left(1-, 8-\mathrm{NHCH}_{2}\right)$, 37.23 (2-, 6-C), 28.23, 24.52 (7-, 9-, 10-C), $21.92\left(3-\right.$, 5-C). IR ( $\left.\mathrm{cm}^{-1}\right)$ : $3175(\mathrm{~m}), 3087$ (m, $v_{\mathrm{N}-\mathrm{H}}$ and $\left.v_{=\mathrm{CH}}\right), 2962(\mathrm{~m}), 2924(\mathrm{~m}), 2855\left(\mathrm{~m}, v_{\mathrm{CH}}\right), 1583\left(\mathrm{~m}, \delta_{\mathrm{N}-\mathrm{H}}\right), 1459(\mathrm{~m}), 1437\left(\mathrm{~m}, \tau_{\mathrm{CH} 2}\right.$ and $\left.v_{\mathrm{C}=\mathrm{C} \text { of aromatic ring }}\right), 1368\left(\mathrm{~m}, \tau_{\mathrm{CH} 3}\right), 1128\left(\mathrm{~m}, v_{\mathrm{C}-\mathrm{N}}\right), 1059\left(\mathrm{~m}, v_{\mathrm{C}-\mathrm{O}}\right), 1032(\mathrm{~m}), 958(\mathrm{~m})$, 
958 (s); HRMS (ESI) for $\mathrm{C}_{20} \mathrm{H}_{33} \mathrm{~N}_{4}$, calcd. 329.2705, found $329.2706[\mathrm{M}+\mathrm{H}]^{+}, \Delta=-1.74$ ppm.

9a, Light yellow oil. ${ }^{1} \mathrm{H}$ NMR (DMSO- $\left.d_{6}, 400 \mathrm{MHz}\right) \delta(\mathrm{ppm}): 7.01 \sim 6.97,6.52 \sim 6.50$, 6.47 6.43 (3m, 5H, Ar-H), $5.68(1 \mathrm{t}, 1 \mathrm{H}, J=6.0 \mathrm{~Hz}, 8-\mathrm{NH}), 5.59(1 \mathrm{~s}, 1 \mathrm{H}, 2-\mathrm{H}), 4.66$ (1s, $2 \mathrm{H}, 10-\mathrm{H}), 3.50\left(1 \mathrm{~d}, 2 \mathrm{H}, J=4.0 \mathrm{~Hz}, 7-\mathrm{CH}_{2}\right), 2.07 \sim 2.04$ (1m, 1H, 4-H), 2.01 1.99 (1m, 3H, 3-, 5-, 6- $\left.\mathrm{H}_{\mathrm{e}}\right), 1.88 \sim 1.80\left(1 \mathrm{~m}, 1 \mathrm{H}, 6-\mathrm{H}_{\mathrm{a}}\right), 1.76 \sim 1.71\left(1 \mathrm{~m}, 1 \mathrm{H}, 3-\mathrm{H}_{\mathrm{a}}\right), 1.66(1 \mathrm{~s}, 3 \mathrm{H}, 9-\mathrm{H})$, $1.42 \sim 1.31\left(1 \mathrm{~m}, 1 \mathrm{H}, 5-\mathrm{H}_{\mathrm{a}}\right) ;{ }^{13} \mathrm{C}$ NMR (DMSO- $\left.d_{6}, 101 \mathrm{MHz}\right) \delta(\mathrm{ppm}): 149.42(8-\mathrm{C}), 135.22$ (1-C), 149.25, 128.84, 120.73, 112.16 (Ar-C), 115.48 (2-C), 108.92 (10-C), 48.78 (7-C), 40.89 (4-C), 30.11 (3-C), 27.36, 26.69 (5-C, 6-C), 20.77 (9-C); IR ( $\left.\mathrm{cm}^{-1}\right): 3416$ (m, $v_{\mathrm{N}-\mathrm{H}}$ ), $3052\left(\mathrm{w}, v_{=\mathrm{CH}}\right), 2917(\mathrm{~m}), 2833\left(\mathrm{~m}, v_{\mathrm{CH}}\right), 1642\left(\mathrm{~m}, \delta_{\mathrm{N}-\mathrm{H}}\right), 1602\left(\mathrm{~s}, \delta_{\mathrm{C}=\mathrm{C}}\right), 1502\left(\mathrm{~s}, \delta_{\mathrm{C}=\mathrm{C} \text { of }}\right.$ aromatic ring $), 1430\left(\mathrm{~m}, \tau_{\mathrm{CH} 2}\right), 1318\left(\mathrm{~m}, \tau_{\mathrm{CH} 3}\right), 1250(\mathrm{~m}), 1179\left(\mathrm{~m}, v_{\mathrm{C}-\mathrm{N}}\right), 883(\mathrm{~m}), 747(\mathrm{~s})$; HRMS (ESI) for $\mathrm{C}_{16} \mathrm{H}_{22} \mathrm{~N}$, calcd. 228.1752, found $228.1772[\mathrm{M}+\mathrm{H}]^{+}, \Delta=-10.86 \mathrm{ppm}$.

9b, Light yellow oil. ${ }^{1} \mathrm{H}$ NMR (DMSO- $\left.d_{6}, 400 \mathrm{MHz}\right) \delta(\mathrm{ppm}): 6.89 \sim 6.85,6.55 \sim 6.51$ (2m, 4H, Ar-H), 5.69 (1t, 1H, J=6.0 Hz, 8-NH), $5.62(1 \mathrm{~m}, 1 \mathrm{H}, 2-\mathrm{H}), 4.70(1 \mathrm{~s}, 2 \mathrm{H}, 10-\mathrm{H})$, $3.50\left(1 \mathrm{~d}, 2 \mathrm{H}, J=4.0 \mathrm{~Hz}, 7-\mathrm{CH}_{2}\right), 2.10 \sim 2.02\left(1 \mathrm{~m}, 4 \mathrm{H}, 4-\mathrm{H}, 3-, 5-, 6-\mathrm{H}_{\mathrm{e}}\right), 1.92 \sim 1.84(1 \mathrm{~m}, 1 \mathrm{H}$, 6- $\left.\mathrm{H}_{\mathrm{a}}\right), 1.79 \sim 1.73\left(1 \mathrm{~m}, 1 \mathrm{H}, 3-\mathrm{H}_{\mathrm{a}}\right), 1.70(1 \mathrm{~s}, 3 \mathrm{H}, 9-\mathrm{H}), 1.45 \sim 1.35\left(1 \mathrm{~m}, 1 \mathrm{H}, 5-\mathrm{H}_{\mathrm{a}}\right) ;{ }^{13} \mathrm{C} \mathrm{NMR}$ $\left(\mathrm{DMSO}-d_{6}, 101 \mathrm{MHz}\right) \delta(\mathrm{ppm}): 153.86$ (8-C), 146.70 (1-C), 156.15, 150.17, 146.69, 135.89, 121.64, 115.86, 113.59, 113.52 (Ar-C), 116.07 (2-C), 109.73 (10-C), 50.01 (7-C), 41.60 (4-C), 30.81 (3-C), 28.06, 27.41 (5-C, 6-C), 21.53 (9-C); IR ( $\left.\mathrm{cm}^{-1}\right): 3423\left(\mathrm{~m}, v_{\mathrm{N}-\mathrm{H}}\right), 3072$ $\left(\mathrm{w}, v_{=\mathrm{CH}}\right), 2917(\mathrm{~m}), 2835\left(\mathrm{~m}, v_{\mathrm{CH}}\right), 1642\left(\mathrm{~m}, \delta_{\mathrm{N}-\mathrm{H}}\right), 1611\left(\mathrm{~s}, \delta_{\mathrm{C}=\mathrm{C}}\right), 1507\left(\mathrm{~s}, \delta_{\mathrm{C}=\mathrm{C} \text { of aromatic }}\right.$ ring $), 1438\left(\mathrm{~m}, \tau_{\mathrm{CH} 2}\right), 1321\left(\mathrm{~m}, \tau_{\mathrm{CH} 3}\right), 1221(\mathrm{~s}), 1155\left(\mathrm{~m}, v_{\mathrm{C}-\mathrm{N}}\right), 885(\mathrm{~m}), 815(\mathrm{~s})$; HRMS (ESI) for $\mathrm{C}_{16} \mathrm{H}_{21} \mathrm{FN}$, calcd. 246.1658, found 246.1658 [M+H] $]^{+}, \Delta=-2.04 \mathrm{ppm}$. 
9c, Light yellow oil. ${ }^{1} \mathrm{H}$ NMR (DMSO- $\left.d_{6}, 400 \mathrm{MHz}\right) \delta$ (ppm): 7.07 7.03, 6.56 6.52 (2m, 4H, Ar-H), $6.01(1 \mathrm{t}, 1 \mathrm{H}, J=6.0 \mathrm{~Hz}, 8-\mathrm{NH}), 5.60(1 \mathrm{~m}, 1 \mathrm{H}, 2-\mathrm{H}), 4.69(1 \mathrm{~s}, 2 \mathrm{H}, 10-\mathrm{H})$, $3.53\left(1 \mathrm{~d}, 2 \mathrm{H}, J=4.0 \mathrm{~Hz}, 7-\mathrm{CH}_{2}\right), 2.09 \sim 2.00\left(1 \mathrm{~m}, 4 \mathrm{H}, 4-\mathrm{H}, 3-, 5-, 6-\mathrm{H}_{\mathrm{e}}\right), 1.91 \sim 1.83(1 \mathrm{~m}, 1 \mathrm{H}$, 6- $\left.\mathrm{H}_{\mathrm{a}}\right), 1.79 \sim 1.75\left(1 \mathrm{~m}, 1 \mathrm{H}, 3-\mathrm{H}_{\mathrm{a}}\right), 1.70(1 \mathrm{~s}, 3 \mathrm{H}, 9-\mathrm{H}), 1.44 \sim 1.34\left(1 \mathrm{~m}, 1 \mathrm{H}, 5-\mathrm{H}_{\mathrm{a}}\right) ;{ }^{13} \mathrm{C}$ NMR $\left(\mathrm{DMSO}-d_{6}, 101 \mathrm{MHz}\right) \delta(\mathrm{ppm}): 150.16(8-\mathrm{C}), 135.58(1-\mathrm{C}), 148.87,129.34,121.71,114.22$ (Ar-C), 119.45 (2-C), 109.76 (10-C), 49.39 (7-C), 41.57 (4-C), 31.67 (3-C), 28.04, 27.35 (5-C, 6-C), 21.54 (9-C); IR ( $\left.\mathrm{cm}^{-1}\right): 3352\left(\mathrm{~m}, v_{\mathrm{N}-\mathrm{H}}\right), 3077$ (w, $\left.v_{=\mathrm{CH}}\right), 2917$ (m), $2833(\mathrm{~m}$, $\left.v_{\mathrm{CH}}\right), 1670\left(\mathrm{~s}, \delta_{\mathrm{N}-\mathrm{H}}\right), 1598\left(\mathrm{~s}, \delta_{\mathrm{C}=\mathrm{C}}\right), 1498\left(\mathrm{~s}, \delta_{\mathrm{C}=\mathrm{C} \text { of aromatic ring }}\right), 1434\left(\mathrm{~m}, \tau_{\mathrm{CH} 2}\right), 1382(\mathrm{~m}$, $\left.\tau_{\mathrm{CH} 3}\right), 1255(\mathrm{~m}), 1175\left(\mathrm{~m}, v_{\mathrm{C}-\mathrm{N}}\right), 1090(\mathrm{~s}), 886(\mathrm{~m}), 815(\mathrm{~s})$; HRMS (ESI) for $\mathrm{C}_{16} \mathrm{H}_{21} \mathrm{CIN}$, calcd. 262.1362, found $262.1371[\mathrm{M}+\mathrm{H}]^{+}, \Delta=-5.16 \mathrm{ppm}$.

9d, Light yellow oil. ${ }^{1} \mathrm{H}$ NMR (DMSO- $d_{6}, 400 \mathrm{MHz}$ ) $\delta$ (ppm): 6.85 6.83, 6.47 6.45 (2m, 4H, Ar-H), $5.60(1 \mathrm{~m}, 1 \mathrm{H}, 2-\mathrm{H}), 5.48$ (1t, $1 \mathrm{H}, J=6.0 \mathrm{~Hz}, 8-\mathrm{NH}), 4.69$ (1s, 2H, 10-H), $3.50\left(1 \mathrm{~d}, 2 \mathrm{H}, J=4.0 \mathrm{~Hz}, 7-\mathrm{CH}_{2}\right), 2.13\left(1 \mathrm{~s}, 3 \mathrm{H}, \mathrm{Ar}-\mathrm{CH}_{3}\right), 2.10 \sim 2.01(1 \mathrm{~m}, 4 \mathrm{H}, 4-\mathrm{H}, 3-$, 5-, 6$\left.\mathrm{H}_{\mathrm{e}}\right), 1.91 \sim 1.83\left(1 \mathrm{~m}, 1 \mathrm{H}, 6-\mathrm{H}_{\mathrm{a}}\right), 1.78 \sim 1.74\left(1 \mathrm{~m}, 1 \mathrm{H}, 3-\mathrm{H}_{\mathrm{a}}\right), 1.69(1 \mathrm{~s}, 3 \mathrm{H}, 9-\mathrm{H}), 1.44 \sim 1.34$ (1m, $\left.1 \mathrm{H}, 5-\mathrm{H}_{\mathrm{a}}\right) ;{ }^{13} \mathrm{C}$ NMR (DMSO- $\left.d_{6}, 101 \mathrm{MHz}\right) \delta(\mathrm{ppm}): 150.19$ (8-C), 136.15 (1-C), 147.75, 130.06, 124.52, 113.04 (Ar-C), 121.39 (2-C), 109.66 (10-C), 49.79 (7-C), 41.65

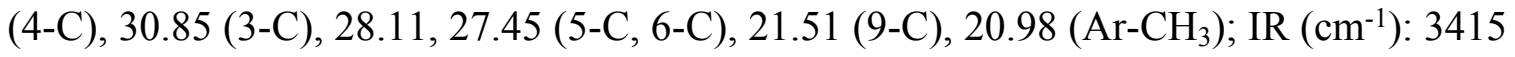
$\left(\mathrm{m}, v_{\mathrm{N}-\mathrm{H}}\right), 3017\left(\mathrm{w}, v_{=\mathrm{CH}}\right), 2917(\mathrm{~m}), 2835\left(\mathrm{~m}, v_{\mathrm{CH}}\right), 1642\left(\mathrm{~m}, \delta_{\mathrm{N}-\mathrm{H}}\right), 1619\left(\mathrm{~m}, \delta_{\mathrm{C}=\mathrm{C}}\right), 1518$ (s, $\left.\delta_{\mathrm{C}=\mathrm{C} \text { of aromatic ring }}\right), 1433\left(\mathrm{~m}, \tau_{\mathrm{CH} 2}\right), 1317\left(\mathrm{~m}, \tau_{\mathrm{CH} 3}\right), 1248(\mathrm{~s}), 1183\left(\mathrm{~m}, v_{\mathrm{C}-\mathrm{N}}\right), 1120(\mathrm{~m})$, 885 (m), 803 (s); HRMS (ESI) for $\mathrm{C}_{17} \mathrm{H}_{24} \mathrm{~N}$, calcd. 242.1909, found $242.1915[\mathrm{M}+\mathrm{H}]^{+}$, $\Delta=-4.69 \mathrm{ppm}$.

9e, Light yellow oil. ${ }^{1} \mathrm{H}$ NMR (DMSO- $\left.d_{6}, 400 \mathrm{MHz}\right) \delta$ (ppm): 6.70 6.66, 6.52 6.48 (2m, 4H, Ar-H), $5.61(1 \mathrm{~s}, 1 \mathrm{H}, 8-\mathrm{NH}), 5.33(1 \mathrm{t}, 1 \mathrm{H}, J=6.0 \mathrm{~Hz}, 2-\mathrm{H}), 4.69$ (1s, 2H, 10-H), 
$3.62\left(1 \mathrm{~s}, 3 \mathrm{H}, \mathrm{Ar}-\mathrm{CH}_{3}\right), 3.48\left(1 \mathrm{~d}, 2 \mathrm{H}, J=8.0 \mathrm{~Hz}, 7-\mathrm{CH}_{2}\right), 2.09 \sim 1.99(1 \mathrm{~m}, 4 \mathrm{H}, 4-\mathrm{H}, 3-$, 5-, 6- $\left.\mathrm{H}_{\mathrm{e}}\right), 1.91 \sim 1.83\left(1 \mathrm{~m}, 1 \mathrm{H}, 6-\mathrm{H}_{\mathrm{a}}\right), 1.78 \sim 1.72\left(1 \mathrm{~m}, 1 \mathrm{H}, 3-\mathrm{H}_{\mathrm{a}}\right), 1.69(1 \mathrm{~s}, 3 \mathrm{H}, 9-\mathrm{H}), 1.44 \sim 1.34$ $\left(1 \mathrm{~m}, 1 \mathrm{H}, 5-\mathrm{H}_{\mathrm{a}}\right) .{ }^{13} \mathrm{C}$ NMR (DMSO- $\left.d_{6}, 101 \mathrm{MHz}\right) \delta(\mathrm{ppm}): 150.64$ (8-C), 143.57 (1-C), 149.52, 135.60, 114.65, 113.18 (Ar-C), 120.70 (2-C), 109.03 (10-C), 55.44 (7-C), 49.62 (4-C), 40.92 (3-C), 30.11 (5-C), 27.38 (6-C), 26.76 (9-C), 20.84 ( $\mathrm{Ar}^{\left.-\mathrm{CH}_{3}\right) .}$ IR ( $\left(\mathrm{cm}^{-1}\right): 3411$ $\left(\mathrm{m}, v_{\mathrm{N}-\mathrm{H}}\right), 3068\left(\mathrm{w}, v_{=\mathrm{CH}}\right), 2913(\mathrm{~m}), 2832\left(\mathrm{~m}, v_{\mathrm{CH}}\right), 1642\left(\mathrm{~m}, \delta_{\mathrm{N}-\mathrm{H}}\right), 1615\left(\mathrm{w}, \delta_{\mathrm{C}=\mathrm{C}}\right), 1511$ $\left(\mathrm{s}, \delta_{\mathrm{C}=\mathrm{C} \text { of aromatic ring }}\right), 1438\left(\mathrm{~m}, \tau_{\mathrm{CH} 2}\right), 1294\left(\mathrm{~m}, \tau_{\mathrm{CH} 3}\right), 1229(\mathrm{~s}), 1179\left(\mathrm{~m}, v_{\mathrm{C}-\mathrm{N}}\right), 885(\mathrm{~m}), 816$ (s); HRMS (ESI) for $\mathrm{C}_{17} \mathrm{H}_{24} \mathrm{NO}$, calcd. 258.1858, found $258.1863[\mathrm{M}+\mathrm{H}]^{+}, \Delta=-4.26 \mathrm{ppm}$. 9f, Light yellow oil. ${ }^{1} \mathrm{H}$ NMR (DMSO- $\left.d_{6}, 400 \mathrm{MHz}\right) \delta$ (ppm): 7.03 7.01, 6.60 6.57 (2m, 4H, Ar-H), $6.11(1 \mathrm{t}, 1 \mathrm{H}, J=6.0 \mathrm{~Hz}, 8-\mathrm{NH}), 5.63(1 \mathrm{~s}, 1 \mathrm{H}, 2-\mathrm{H}), 4.70(1 \mathrm{~s}, 2 \mathrm{H}, 10-\mathrm{H})$, $3.55\left(1 \mathrm{~d}, 2 \mathrm{H}, J=4.0 \mathrm{~Hz}, 7-\mathrm{CH}_{2}\right), 2.11 \sim 1.99\left(1 \mathrm{~m}, 4 \mathrm{H}, 4-\mathrm{H}, 3-, 5-, 6-\mathrm{H}_{\mathrm{e}}\right), 1.92 \sim 1.85(1 \mathrm{~m}, 1 \mathrm{H}$, 6- $\left.\mathrm{H}_{\mathrm{a}}\right), 1.80 \sim 1.77\left(1 \mathrm{~m}, 1 \mathrm{H}, 3-\mathrm{H}_{\mathrm{a}}\right), 1.70(1 \mathrm{~s}, 3 \mathrm{H}, 9-\mathrm{H}), 1.46 \sim 1.36\left(1 \mathrm{~m}, 1 \mathrm{H}, 5-\mathrm{H}_{\mathrm{a}}\right) .{ }^{13} \mathrm{C}$ NMR $\left(\mathrm{DMSO}-d_{6}, 101 \mathrm{MHz}\right) \delta(\mathrm{ppm}): 149.42,138.33,134.83,112.38$ (Ar-C), 148.52 (8-C), 138.35 (1-C), 122.08 ( $\mathrm{Ar}^{-\mathrm{CF}_{3}}$ ), 121.00 (2-C), 108.97 (10-C), 48.79 (7-C), 40.86 (4-C), 30.08 (3-C), 27.32 (5-C), 26.64 (6-C), 20.74 (9-C). IR ( $\left.\mathrm{cm}^{-1}\right)$ : 3427 (m, $\left.v_{\mathrm{N}-\mathrm{H}}\right), 3075$ (w, $\left.v_{=\mathrm{CH}}\right), 2917(\mathrm{~m}), 2837\left(\mathrm{~m}, v_{\mathrm{CH}}\right), 1642\left(\mathrm{~m}, \delta_{\mathrm{N}-\mathrm{H}}\right), 1611\left(\mathrm{~s}, \delta_{\mathrm{C}=\mathrm{C}}\right), 1515\left(\mathrm{~s}, \delta_{\mathrm{C}=\mathrm{C} \text { of aromatic ring }}\right)$, $1439\left(\mathrm{~m}, \delta_{\mathrm{N}-\mathrm{H}}, \tau_{\mathrm{CH} 2}\right), 1246\left(\mathrm{~s}, \tau_{\mathrm{CH} 3}\right), 1222(\mathrm{~s}), 1198\left(\mathrm{w}, v_{\mathrm{C}-\mathrm{N}}\right), 887(\mathrm{~m}), 831(\mathrm{~m})$; HRMS (ESI) for $\mathrm{C}_{17} \mathrm{H}_{21} \mathrm{~F}_{3} \mathrm{NO}$, calcd. 312.1575, found $312.1579[\mathrm{M}+\mathrm{H}]^{+}, \Delta=-2.78 \mathrm{ppm}$.

9g, Light yellow oil. ${ }^{1} \mathrm{H}$ NMR (DMSO- $\left.d_{6}, 400 \mathrm{MHz}\right) \delta(\mathrm{ppm})$ : 7.22 7.20, 7.09 7.05, 6.60 6.51 (3m, 4H, Ar-H), 5.56 5.53 (1m, 2H, 8-NH, 2-H), 4.68 (1s, 2H, 10-H), 3.68 (1d, $\left.2 \mathrm{H}, J=8.0 \mathrm{~Hz}, 7-\mathrm{CH}_{2}\right), 2.08 \sim 1.99\left(1 \mathrm{~m}, 4 \mathrm{H}, 4-\mathrm{H}, 3-, 5-, 6-\mathrm{H}_{\mathrm{e}}\right), 1.90 \sim 1.82\left(1 \mathrm{~m}, 1 \mathrm{H}, 6-\mathrm{H}_{\mathrm{a}}\right)$, 1.78 1.74 (1m, 1H, 3- $\left.\mathrm{H}_{\mathrm{a}}\right), 1.68(1 \mathrm{~s}, 3 \mathrm{H}, 9-\mathrm{H}), 1.44 \sim 1.34\left(1 \mathrm{~m}, 1 \mathrm{H}, 5-\mathrm{H}_{\mathrm{a}}\right) .{ }^{13} \mathrm{C}$ NMR $\left(\mathrm{DMSO}-d_{6}, 101 \mathrm{MHz}\right) \delta(\mathrm{ppm}): 144.39,128.93,127.94,120.61,117.69,111.61$ (Ar-C), 
149.34 (8-C), 134.45 (1-C), 116.27 (2-C), 108.96 (10-C), 48.27 (7-C), 40.82 (4-C), 30.02 (3-C), $27.24(5-\mathrm{C}), 26.40$ (6-C), $20.76(9-\mathrm{C}) . \mathrm{IR}\left(\mathrm{cm}^{-1}\right)$ : 3427 (m, $\left.v_{\mathrm{N}-\mathrm{H}}\right), 3072\left(\mathrm{w}, v_{=\mathrm{CH}}\right)$, $2917(\mathrm{~m}), 2835\left(\mathrm{~m}, v_{\mathrm{CH}}\right), 1642\left(\mathrm{~m}, \delta_{\mathrm{N}-\mathrm{H}}\right), 1595\left(\mathrm{~s}, \delta_{\mathrm{C}=\mathrm{C}}\right), 1511\left(\mathrm{~s}, \delta_{\mathrm{C}=\mathrm{C} \text { of aromatic ring }}\right), 1457$ $\left(\mathrm{m}, \tau_{\mathrm{CH} 2}\right), 1321\left(\mathrm{~m}, \tau_{\mathrm{CH} 3}\right), 1031\left(\mathrm{~s}, v_{\mathrm{C}-\mathrm{N}}\right), 885(\mathrm{~s}), 738(\mathrm{~s})$; HRMS (ESI) for $\mathrm{C}_{16} \mathrm{H}_{21} \mathrm{BrN}$, calcd. 306.0857, found $306.0873[\mathrm{M}+\mathrm{H}]^{+}, \Delta=-6.72 \mathrm{ppm}$.

9h, Light yellow oil. ${ }^{1} \mathrm{H}$ NMR (DMSO- $\left.d_{6}, 400 \mathrm{MHz}\right) \delta$ (ppm): 7.06 7.00, 7.39 7.36, 6.30 6.22 (4m, 4H, Ar-H), 6.15 (1t, 1H, $J=6.0 \mathrm{~Hz}, 8-\mathrm{NH}), 5.62$ (1t, $1 \mathrm{H}, J=4.0 \mathrm{~Hz}, 2-\mathrm{H})$, $4.70(1 \mathrm{~s}, 2 \mathrm{H}, 10-\mathrm{H}), 3.54\left(1 \mathrm{~d}, 2 \mathrm{H}, J=8.0 \mathrm{~Hz}, 7-\mathrm{CH}_{2}\right), 2.10 \sim 2.01(1 \mathrm{~m}, 4 \mathrm{H}, 4-\mathrm{H}, 3-, 5-, 6-$ $\left.\mathrm{H}_{\mathrm{e}}\right), 1.92 \sim 1.87\left(1 \mathrm{~m}, 1 \mathrm{H}, 6-\mathrm{H}_{\mathrm{a}}\right), 1.80 \sim 1.76\left(1 \mathrm{~m}, 1 \mathrm{H}, 3-\mathrm{H}_{\mathrm{a}}\right), 1.70(1 \mathrm{~s}, 3 \mathrm{H}, 9-\mathrm{H}), 1.45 \sim 1.35$ $\left(1 \mathrm{~m}, 1 \mathrm{H}, 5-\mathrm{H}_{\mathrm{a}}\right) ;{ }^{13} \mathrm{C}$ NMR (DMSO- $\left.d_{6}, 101 \mathrm{MHz}\right) \delta(\mathrm{ppm}): 163.19$ (8-C), 150.16 (1-C), 135.58 (2-C), 165.56, 152.12, 152.01, 131.01, 130.91, 121.76, 109.80, 109.27, 102.31, 99.06, 98.82 (Ar-C), 102.09 (10-C), 49.28 (7-C), 41.54 (4-C), 30.77 (3-C), 28.03 (5-C), $27.33(6-\mathrm{C}), 21.57$ (9-C). IR $\left(\mathrm{cm}^{-1}\right): 3424\left(\mathrm{~m}, v_{\mathrm{N}-\mathrm{H}}\right), 3073\left(\mathrm{w}, v_{=\mathrm{CH}}\right), 2917,2837\left(\mathrm{~m}, v_{\mathrm{CH}}\right)$, $1642\left(\mathrm{~m}, \delta_{\mathrm{N}-\mathrm{H}}\right), 1618\left(\mathrm{~s}, \delta_{\mathrm{C}=\mathrm{C}}\right), 1586(\mathrm{~s}), 1507\left(\mathrm{~s}, \delta_{\mathrm{C}=\mathrm{C} \text { of aromatic ring }}\right), 1434\left(\mathrm{~m}, \delta_{\mathrm{N}-\mathrm{H}}, \tau_{\mathrm{CH} 2}\right)$, $1334\left(\mathrm{~m}, \tau_{\mathrm{CH} 3}\right), 1252(\mathrm{~m}), 1175\left(\mathrm{~m}, v_{\mathrm{C}-\mathrm{N}}\right), 885$ (s), 755 (s); HRMS (ESI) for $\mathrm{C}_{16} \mathrm{H}_{21} \mathrm{ClN}$, calcd. 262.1362, found $262.1370[\mathrm{M}+\mathrm{H}]^{+}, \Delta=-4.97 \mathrm{ppm}$.

9i, Light yellow oil. ${ }^{1} \mathrm{H}$ NMR (DMSO- $\left.d_{6}, 400 \mathrm{MHz}\right) \delta$ (ppm): 6.98 6.94, 6.50 6.49, 6.45 6.42 (3m, 4H, Ar-H), 6.08 (1t, 1H, $J=6.0 \mathrm{~Hz}, 8-\mathrm{NH}), 5.55(1 \mathrm{~s}, 1 \mathrm{H}, 2-\mathrm{H}), 4.64(1 \mathrm{~s}$, 2H, 10-H), $3.48\left(1 \mathrm{~d}, 2 \mathrm{H}, J=8.0 \mathrm{~Hz}, 7-\mathrm{CH}_{2}\right), 2.04 \sim 1.93\left(1 \mathrm{~m}, 4 \mathrm{H}, 4-\mathrm{H}, 3-, 5-, 6-\mathrm{H}_{\mathrm{e}}\right)$, $1.86 \sim 1.78\left(1 \mathrm{~m}, 1 \mathrm{H}, 6-\mathrm{H}_{\mathrm{a}}\right), 1.73 \sim 1.69\left(1 \mathrm{~m}, 1 \mathrm{H}, 3-\mathrm{H}_{\mathrm{a}}\right), 1.64(1 \mathrm{~s}, 3 \mathrm{H}, 9-\mathrm{H}), 1.39 \sim 1.29(1 \mathrm{~m}$, $\left.1 \mathrm{H}, 5-\mathrm{H}_{\mathrm{a}}\right) .{ }^{13} \mathrm{C}$ NMR (DMSO- $\left.d_{6}, 101 \mathrm{MHz}\right) \delta(\mathrm{ppm}): 149.33,133.73,130.32,114.87$, 113.35, 110.75 (Ar-C), 150.70 (8-C), 134.68 (1-C), 121.04 (2-C), 109.00 (10-C), 48.46 (7C), 40.80 (4-C), 30.06 (3-C), 27.28 (5-C), 26.58 (6-C), 20.79 (9-C); IR ( $\left.\mathrm{cm}^{-1}\right)$ : 3422 (m, 
$\left.v_{\mathrm{N}-\mathrm{H}}\right), 3072\left(\mathrm{w}, v_{=\mathrm{CH}}\right), 2917(\mathrm{~m}), 2835\left(\mathrm{~m}, v_{\mathrm{CH}}\right), 1642\left(\mathrm{~m}, \delta_{\mathrm{N}-\mathrm{H}}\right), 1596\left(\mathrm{~s}, \delta_{\mathrm{C}=\mathrm{C} \text { of aromatic ring }}\right)$, $1499\left(\mathrm{~s}, \delta_{\mathrm{C}=\mathrm{C} \text { of aromatic ring }}\right), 1434\left(\mathrm{~m}, \tau_{\mathrm{CH} 2}\right), 1321\left(\mathrm{~m}, \tau_{\mathrm{CH} 3}\right), 1252(\mathrm{~m}), 1078\left(\mathrm{~m}, v_{\mathrm{C}-\mathrm{N}}\right), 981$ (m), 885 (m), 761 (s); HRMS (ESI) for $\mathrm{C}_{16} \mathrm{H}_{21} \mathrm{BrN}$, calcd. 306.0857, found 306.0859 $[\mathrm{M}+\mathrm{H}]^{+}, \Delta=-2.30 \mathrm{ppm}$.

9j, Light yellow oil. ${ }^{1} \mathrm{H}$ NMR (DMSO- $\left.d_{6}, 400 \mathrm{MHz}\right) \delta(\mathrm{ppm}): 6.90 \sim 6.86,6.34 \sim 6.28$ $(2 \mathrm{~m}, 4 \mathrm{H}, \mathrm{Ar}-\mathrm{H}), 5.58 \sim 5.55(1 \mathrm{~m}, 2 \mathrm{H}, 8-\mathrm{NH}, 2-\mathrm{H}), 4.66(1 \mathrm{~s}, 2 \mathrm{H}, 10-\mathrm{H}), 3.49(1 \mathrm{~d}, 2 \mathrm{H}, J=4.0$ $\left.\mathrm{Hz}, 7-\mathrm{CH}_{2}\right), 2.13\left(1 \mathrm{~m}, 3 \mathrm{H}, \mathrm{Ar}-\mathrm{CH}_{3}\right), 2.05 \sim 1.98\left(1 \mathrm{~m}, 4 \mathrm{H}, 4-\mathrm{H}, 3-\right.$, 5-, 6- $\left.\mathrm{H}_{\mathrm{e}}\right), 1.89 \sim 1.81(1 \mathrm{~m}$, $\left.1 \mathrm{H}, 6-\mathrm{H}_{\mathrm{a}}\right), 1.76 \sim 1.72\left(1 \mathrm{~m}, 1 \mathrm{H}, 3-\mathrm{H}_{\mathrm{a}}\right), 1.66(1 \mathrm{~s}, 3 \mathrm{H}, 9-\mathrm{H}), 1.42 \sim 1.32\left(1 \mathrm{~m}, 1 \mathrm{H}, 5-\mathrm{H}_{\mathrm{a}}\right) .{ }^{13} \mathrm{C}$ NMR (DMSO- $\left.d_{6}, 101 \mathrm{MHz}\right) \delta(\mathrm{ppm}): 149.28,135.36,128.81,116.49,112.92,109.36$ (ArC), 149.47 (8-C), 137.77 (1-C), 120.66 (2-C), 109.04 (10-C), 48.72 (7-C), 40.86 (4-C),

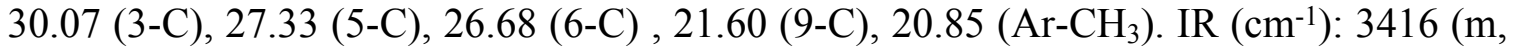
$\left.v_{\mathrm{N}-\mathrm{H}}\right), 3079\left(\mathrm{w}, v_{=\mathrm{CH}}\right), 2917(\mathrm{~m}), 2835\left(\mathrm{~m}, v_{\mathrm{CH}}\right), 1642\left(\mathrm{~m}, \delta_{\mathrm{N}-\mathrm{H}}\right), 1604\left(\mathrm{~s}, \delta_{\mathrm{C}=\mathrm{C}}\right), 1507(\mathrm{~s}$, $\left.\delta_{\mathrm{C}=\mathrm{C} \text { of aromatic ring }}\right), 1433\left(\mathrm{~m}, \tau_{\mathrm{CH} 2}\right), 1325\left(\mathrm{~m}, \tau_{\mathrm{CH} 3}\right), 1178\left(\mathrm{~m}, v_{\mathrm{C}-\mathrm{N}}\right), 989(\mathrm{~m}), 885(\mathrm{~m}), 765(\mathrm{~s})$; HRMS (ESI) for $\mathrm{C}_{16} \mathrm{H}_{28} \mathrm{~N}$, calcd. 234.2222, found $234.2225[\mathrm{M}+\mathrm{H}]^{+}, \Delta=-3.55 \mathrm{ppm}$.

9k, Light yellow oil. ${ }^{1} \mathrm{H}$ NMR (DMSO- $\left.d_{6}, 400 \mathrm{MHz}\right) \delta(\mathrm{ppm}): 5.54$ (1m, 1H, 2-H), $4.70(1 \mathrm{~s}, 2 \mathrm{H}, 10-\mathrm{H}), 3.04\left(1 \mathrm{~d}, 2 \mathrm{H}, J=14.0 \mathrm{~Hz}, 7-\mathrm{NHCH}_{2}\right), 2.33 \sim 2.26(1 \mathrm{~m}, 1 \mathrm{H}, 1$ '-H), 2.10 2.02 (1m, 4H, 4-H, 3-, 5-, 6- $\left.\mathrm{H}_{\mathrm{e}}\right), 1.91 \sim 1.63$ (2m, 5H, 3-, 6- $\mathrm{H}_{\mathrm{a}}, \mathrm{NH}, 2$ '-, 6'- $\left.\mathrm{H}_{\mathrm{e}}\right), 1.70$ (1s, 3H, 9-H), 1.67 1.63 (1m, 2H, 4'- $\left.-\mathrm{H}_{\mathrm{e}}, 4^{\prime}-\mathrm{H}_{\mathrm{a}}\right), 1.55 \sim 1.51\left(1 \mathrm{~m}, 1 \mathrm{H}, 5-\mathrm{H}_{\mathrm{a}}\right), 1.43 \sim 1.33(1 \mathrm{~m}$, $\left.1 \mathrm{H}, 2^{\prime}-\mathrm{H}_{\mathrm{a}}\right), 1.23 \sim 1.15\left(1 \mathrm{~m}, 5 \mathrm{H}, 6^{\prime}-\mathrm{H}_{\mathrm{a}}, 3^{\prime}-, 5^{\prime}-\mathrm{H}_{\mathrm{e}}, 3^{\prime}-, 5^{\prime}-\mathrm{H}_{\mathrm{a}}\right) .{ }^{13} \mathrm{C}$ NMR (DMSO-d 6,101 MHz) $\delta$ (ppm): 150.29 (8-C), 137.80 (1-C), 120.87, 121.32 (2-C), 109.60 (10-C), 56.19 (1'-C), 53.13 (7-C), 41.68 (4-C), 33.99, 33.87 (2'-, 6'-C), 30.92 (3-C), 28.20, 27.81 (5-, 6C), 26.87 (4'-C), 25.40 (3'-, 5'-C), 21.53 (9-C); IR (cm-1): 3076 (w, v=cH), 2925 (m), 2850 
$\left(\mathrm{m}, v_{\mathrm{CH}}\right), 1642\left(\mathrm{~m}, \delta_{\mathrm{N}-\mathrm{H}}\right), 1449\left(\mathrm{~m}, \tau_{\mathrm{CH} 2}\right), 1372\left(\mathrm{~m}, \tau_{\mathrm{CH} 3}\right), 1115\left(\mathrm{~m}, v_{\mathrm{C}-\mathrm{N}}\right), 885(\mathrm{~s}), 724(\mathrm{~m})$; HRMS (ESI) for $\mathrm{C}_{17} \mathrm{H}_{30} \mathrm{~N}$, calcd. 248.2378, found $248.2390[\mathrm{M}+\mathrm{H}]^{+}, \Delta=-7.13 \mathrm{ppm}$.

91, Light yellow oil. ${ }^{1} \mathrm{H}$ NMR (DMSO- $\left.d_{6}, 400 \mathrm{MHz}\right) \delta(\mathrm{ppm}): 5.54$ (1s, $\left.1 \mathrm{H}, 2-\mathrm{H}\right), 4.70$ (1s, 2H, 10-H), 3.11, 2.98 (1d, 2H, J=14.0 Hz, 7- $\mathrm{NHCH}_{2}$ ), 2.08 2.05 (1m, 4H, 4-H, 3-, 5-, 6- $\left.\mathrm{H}_{\mathrm{e}}\right), 1.96 \sim 1.83\left(1 \mathrm{~m}, 3 \mathrm{H}, 6-\mathrm{H}_{\mathrm{a}}, \mathrm{NH}, 1\right.$ '-H), 1.78 1.74 (1m, 1H, 3- $\left.\mathrm{H}_{\mathrm{a}}\right), 1.70(1 \mathrm{~s}, 3 \mathrm{H}, 9-\mathrm{H})$, 1.65 1.62, 1.57 1.55 (2m, 3H, 3'-, 4'-, 6'- $\left.-\mathrm{H}_{\mathrm{a}}\right), 1.42 \sim 1.32\left(1 \mathrm{~m}, 2 \mathrm{H}, 5-\mathrm{H}_{\mathrm{a}}, 2^{\prime}-\mathrm{H}\right), 1.24 \sim 1.09$ (1m, 4H, 3'-, 4'-, 6'- $\left.\mathrm{H}_{\mathrm{e}}, 5^{\prime}-\mathrm{H}_{\mathrm{a}}\right), 0.99 \sim 0.87\left(1 \mathrm{~m}, 4 \mathrm{H}, 5^{\prime}-\mathrm{H}_{\mathrm{e}}, 2^{\prime}-\mathrm{CH}_{3}\right) \cdot{ }^{13} \mathrm{C}$ NMR (DMSO- $d_{6}$, $101 \mathrm{MHz}) \delta(\mathrm{ppm}): 149.60,149.54$ (8-C), 137.24 (1-C), 120.87, 120.75 (2-C), 108.96 (10C), 61.32, 61.08 (1'-C), 52.51, 52.15 (7-C), 40.96 (4-C), 37.88, 37.81 (2'-C), 34.49 (3'-C), 32.05, 31.87 (6'-C), 30.23, 30.19 (3-C), 27.48, 27.44 (3'-, 6'-C), 27.16 (5-C), 26.87 (6-C), 25.80 (4'-C), 25.42 (5'-C), 20.88, 20.85 (9-C), 19.74, $19.71\left(2^{\prime}-\mathrm{CH}_{3}\right)$. IR ( $\left.\mathrm{cm}^{-1}\right)$ : 3083 (w, $\left.v_{\mathrm{CH}}\right), 2920(\mathrm{~m}), 2854\left(\mathrm{~m}, v_{\mathrm{CH}}\right), 1642\left(\mathrm{~m}, \delta_{\mathrm{N}-\mathrm{H}}\right), 1448\left(\mathrm{~m}, \tau_{\mathrm{CH} 2}\right), 1375\left(\mathrm{~m}, \tau_{\mathrm{CH} 3}\right), 1115(\mathrm{~m}$, $\left.v_{\mathrm{C}-\mathrm{N}}\right), 887(\mathrm{~s}), 714(\mathrm{~m})$; HRMS (ESI) for $\mathrm{C}_{14} \mathrm{H}_{26} \mathrm{~N}$, calcd. 208.2065, found 208.2069 $[\mathrm{M}+\mathrm{H}]^{+}, \Delta=-4.59 \mathrm{ppm}$.

9m, Light yellow oil. ${ }^{1} \mathrm{H}$ NMR ( $\left.\mathrm{CDCl}_{3}, 400 \mathrm{MHz}\right) \delta$ (ppm): 5.53 (1s, 1H, 2-H), 4.69 (1s, 2H, 10-H), $3.00\left(1 \mathrm{~s}, 2 \mathrm{H}, 7-\mathrm{CH}_{2}\right), 2.41(1 \mathrm{t}, 2 \mathrm{H}, J=8.0 \mathrm{~Hz}, 7-n$-butyl-NHCH 2$), 2.10 \sim 2.02$ (1m, 4H, 4-H, 3-, 5-, 6- $\left.\mathrm{H}_{\mathrm{e}}\right), 1.99(1 \mathrm{~s}, 1 \mathrm{H}, \mathrm{NH}), 1.92 \sim 1.84\left(1 \mathrm{~m}, 1 \mathrm{H}, 6-\mathrm{H}_{\mathrm{a}}\right), 1.79 \sim 1.73(1 \mathrm{~m}$, $\left.1 \mathrm{H}, 3-\mathrm{H}_{\mathrm{a}}\right), 1.70(1 \mathrm{~s}, 3 \mathrm{H}, 9-\mathrm{H}), 1.43 \sim 1.25\left(1 \mathrm{~m}, 3 \mathrm{H}, 5-\mathrm{H}_{\mathrm{a}}, 7-n\right.$-butyl- $\left.\mathrm{NHCH}_{2}\left(\mathrm{CH}_{2}\right)_{2}\right), 0.86$ (1t, 3H, $J=6.0 \mathrm{~Hz}, 7-n$-butyl-CH$\left.{ }_{3}\right) .{ }^{13} \mathrm{C}$ NMR (DMSO- $\left.d_{6}, 101 \mathrm{MHz}\right) \delta(\mathrm{ppm}): 149.81$ (8C), 136.95 (1-C), 121.18 (2-C), 109.11 (10-C), 55.75 (7-C), 48.69 (7-n-butyl- $\mathrm{NHCH}_{2}$ ), 41.25 (4-C), 32.26 (n-butyl- $\mathrm{CH}_{2} \mathrm{CH}_{2}$ ), 30.48 (3-C), 27.74 (5-C), 27.23 (6-C), 21.05 (9-C), 20.50 (7-n-butyl- $\mathrm{CH}_{2} \mathrm{CH}_{3}$ ), 14.38 (7-n-butyl- $\mathrm{CH}_{3}$ ). IR ( $\left.\mathrm{cm}^{-1}\right)$ : 3083 (w, $\left.v_{=\mathrm{CH}}\right), 2958,2921$, $2855\left(\mathrm{~m}, v_{\mathrm{CH}}\right), 1642\left(\mathrm{~m}, \delta_{\mathrm{N}-\mathrm{H}}\right), 1453(\mathrm{~m}), 1434\left(\mathrm{~m}, \tau_{\mathrm{CH} 2}\right), 1372\left(\mathrm{~m}, \tau_{\mathrm{CH} 3}\right), 1112\left(\mathrm{~m}, v_{\mathrm{C}-\mathrm{N}}\right)$, 
887 (s), 731 (m); HRMS (ESI) for $\mathrm{C}_{15} \mathrm{H}_{28} \mathrm{~N}$, calcd. 222.2222, found $222.2232[\mathrm{M}+\mathrm{H}]^{+}$, $\Delta=-7.08 \mathrm{ppm}$.

9n, Light yellow oil. $\delta$ (ppm): 5.53 (1s, 1H, 2-H), 4.69 (1s, 2H, 10-H), 2.99 (1s, 2H, 7- $\left.\mathrm{CH}_{2}\right), 2.40\left(1 \mathrm{t}, 2 \mathrm{H}, J=6.0 \mathrm{~Hz}, 7-n\right.$-pentane- $\left.\mathrm{NHCH}_{2}\right), 2.09 \sim 2.01(1 \mathrm{~m}, 4 \mathrm{H}, 4-\mathrm{H}, 3-$, 5-, 6$\left.\mathrm{H}_{\mathrm{e}}\right), 1.99(1 \mathrm{~s}, 1 \mathrm{H}, \mathrm{NH}), 1.92 \sim 1.83\left(1 \mathrm{~m}, 1 \mathrm{H}, 6-\mathrm{H}_{\mathrm{a}}\right), 1.79 \sim 1.72\left(1 \mathrm{~m}, 1 \mathrm{H}, 3-\mathrm{H}_{\mathrm{a}}\right), 1.70(1 \mathrm{~s}$, $3 \mathrm{H}, 9-\mathrm{H}), 1.41 \sim 1.34\left(1 \mathrm{~m}, 3 \mathrm{H}, 5-\mathrm{H}_{\mathrm{a}}, 7-n-\right.$ pentane- $\left.\mathrm{NHCH}_{2} \mathrm{CH}_{2}\right), 1.27 \sim 1.24(1 \mathrm{~m}, 4 \mathrm{H}, 7-n-$ pentane- $\left.\mathrm{NH}\left(\mathrm{CH}_{2}\right)_{2}\left(\mathrm{CH}_{2}\right)_{2}\right), 0.86(1 \mathrm{t}, 3 \mathrm{H}, J=8.0 \mathrm{~Hz}, 7-n$-pentane-CH$) .{ }^{13} \mathrm{C}$ NMR (DMSO$\left.d_{6}, 101 \mathrm{MHz}\right) \delta(\mathrm{ppm}): 149.53$ (8-C), 136.67 (1-C), 120.88 (2-C), 108.84 (10-C), 55.44 (7C), 48.72 (7-n-pentane- $\mathrm{NHCH}_{2}$ ), 40.94 (4-C), 30.17 (n-pentane- $\mathrm{CH}_{2}-\mathrm{CH}_{2}$ ), 29.45 (3-C), 29.33 (7-n-hexane- $\mathrm{NH}\left(\mathrm{CH}_{2}\right)_{2} \mathrm{CH}_{2}$ ), 27.44 (5-C), 26.93 (6-C), 22.31 (7-n-hexane- $\mathrm{CH}_{2} \mathrm{CH}_{3}$ ), 20.77 (9-C), 14.12 (7-n-hexane- $\left.\mathrm{CH}_{3}\right)$. IR $\left(\mathrm{cm}^{-1}\right)$ : 3079 (w, $\left.v_{=\mathrm{CH}}\right), 2954(\mathrm{~m}), 2920(\mathrm{~m}), 2857$ $\left(\mathrm{m}, v_{\mathrm{CH}}\right), 1645\left(\mathrm{~m}, \delta_{\mathrm{N}-\mathrm{H}}\right), 1451(\mathrm{~m}), 1434\left(\mathrm{~m}, \tau_{\mathrm{CH} 2}\right), 1375\left(\mathrm{~m}, \tau_{\mathrm{CH} 3}\right), 1109\left(\mathrm{~m}, v_{\mathrm{C}-\mathrm{N}}\right), 887(\mathrm{~s})$, 727 (m); HRMS (ESI) for $\mathrm{C}_{16} \mathrm{H}_{30} \mathrm{~N}$, calcd. 236.2378, found $236.2372[\mathrm{M}+\mathrm{H}]^{+}, \Delta=0.40$ ppm.

9o, Light yellow oil. ${ }^{1} \mathrm{H}$ NMR (DMSO- $\left.d_{6}, 400 \mathrm{MHz}\right) \delta$ (ppm): 5.53 (1s, $\left.1 \mathrm{H}, 2-\mathrm{H}\right), 4.70$ $(1 \mathrm{~s}, 2 \mathrm{H}, 10-\mathrm{H}), 2.99\left(1 \mathrm{~s}, 2 \mathrm{H}, 7-\mathrm{CH}_{2}\right), 2.39\left(1 \mathrm{t}, 2 \mathrm{H}, J=6.0 \mathrm{~Hz}, 7-n\right.$-hexane-NHCH$\left.H_{2}\right)$, 2.09 1.98 (1m, 4H, 4-H, 3-, 5-, 6- $\left.\mathrm{H}_{\mathrm{e}}\right), 1.99(1 \mathrm{~s}, 1 \mathrm{H}, \mathrm{NH}), 1.92 \sim 1.83\left(1 \mathrm{~m}, 1 \mathrm{H}, 6-\mathrm{H}_{\mathrm{a}}\right)$, 1.78 1.72 (1m, 1H, 3- $\left.\mathrm{H}_{\mathrm{a}}\right), 1.70(1 \mathrm{~s}, 3 \mathrm{H}, 9-\mathrm{H}), 1.42 \sim 1.34\left(1 \mathrm{~m}, 3 \mathrm{H}, 5-\mathrm{H}_{\mathrm{a}}, 7-n\right.$-hexane$\left.\mathrm{NHCH}_{2} \mathrm{CH}_{2}\right), 1.31 \sim 1.21\left(1 \mathrm{~m}, 6 \mathrm{H}, 7-n\right.$-hexane-NH$\left.\left(\mathrm{CH}_{2}\right)_{2}\left(\mathrm{CH}_{2}\right)_{3}\right), 0.86(1 \mathrm{t}, 3 \mathrm{H}, J=8.0 \mathrm{~Hz}$, 7-n-hexane-CH$\left.H_{3}\right) .{ }^{13} \mathrm{C}$ NMR (DMSO- $\left.d_{6}, 101 \mathrm{MHz}\right) \delta(\mathrm{ppm}): 149.57$ (8-C), 136.69 (1-C), 120.96 (2-C), 108.94 (10-C), 55.45 (7-C), 48.74 (7-n-hexane- $\mathrm{NHCH}_{2}$ ), 40.95 (4-C), 31.53 (n-hexane- $\mathrm{CH}_{2} \mathrm{CH}_{2} \mathrm{CH}_{3}$ ), 30.19 (3-C), 29.73 (7-n-hexane- $\mathrm{NHCH}_{2} \mathrm{CH}_{2}$ ), 27.45 (5-C), 26.95 (6-C), 26.79 (7-n-hexane- $\left.\mathrm{NH}\left(\mathrm{CH}_{2}\right)_{2} \mathrm{CH}_{2}\right), 22.36$ (7-n-hexane- $\mathrm{CH}_{2} \mathrm{CH}_{3}$ ), 20.84 (9-C), 14.17 
(7- $n$-hexane- $\left.\mathrm{CH}_{3}\right)$. IR (cm-1): $3083\left(\mathrm{~m}, v_{=\mathrm{CH}}\right), 2958(\mathrm{~m}), 2923(\mathrm{~m}), 2857\left(\mathrm{~m}, v_{\mathrm{CH}}\right), 1645(\mathrm{~m}$, $\left.\delta_{\mathrm{N}-\mathrm{H}}\right), 1451(\mathrm{~m}), 1434\left(\mathrm{~m}, \tau_{\mathrm{CH} 2}\right), 1375\left(\mathrm{~m}, \tau_{\mathrm{CH} 3}\right), 1109\left(\mathrm{~m}, v_{\mathrm{C}-\mathrm{N}}\right), 887(\mathrm{~s}), 724(\mathrm{~m}) ; \mathrm{HRMS}$ (ESI) for $\mathrm{C}_{16} \mathrm{H}_{30} \mathrm{~N}$, calcd. 236.2378, found $236.2372[\mathrm{M}+\mathrm{H}]^{+}, \Delta=0.40 \mathrm{ppm}$.

Table S1. Inhibition efficacy of hydrogenated cis-p-menthane type Schiff bases against shoot growth of barnyardgrass.

\begin{tabular}{cccccc}
\hline Compd. & Yield $(\%)$ & m.p. $\left({ }^{\circ} \mathrm{C}\right)$ & Compd. & Yield $(\%)$ & m.p. $\left({ }^{\circ} \mathrm{C}\right)$ \\
\hline $\mathbf{6 a}$ & 98 & $66.2 \sim 69.7$ & $\mathbf{9 a}$ & 89 & viscous oil \\
$\mathbf{6 b}$ & 85 & $61.3 \sim 61.7$ & $\mathbf{9 b}$ & 76 & viscous oil \\
$\mathbf{6 c}$ & 96 & $75.1 \sim 75.5$ & $\mathbf{9 c}$ & 82 & viscous oil \\
$\mathbf{6 d}$ & 97 & $48.0 \sim 49.1$ & $\mathbf{9 d}$ & 95 & viscous oil \\
$\mathbf{6 e}$ & 94 & $71.8 \sim 71.9$ & $\mathbf{9 e}$ & 81 & viscous oil \\
$\mathbf{6 f}$ & 96 & $52.1 \sim 52.5$ & $\mathbf{9 f}$ & 82 & viscous oil \\
$\mathbf{6 g}$ & 96 & $105.6 \sim 106.7$ & $\mathbf{9 g}$ & 83 & viscous oil \\
$\mathbf{6 h}$ & 89 & $67.3 \sim 67.4$ & $\mathbf{9 h}$ & 88 & viscous oil \\
$\mathbf{6 i}$ & 83 & $61.9 \sim 62.6$ & $\mathbf{9 i}$ & 63 & viscous oil \\
$\mathbf{6 j}$ & 88 & viscous oil & $\mathbf{9 j}$ & 79 & viscous oil \\
$\mathbf{6 k}$ & 90 & viscous oil & $\mathbf{9 k}$ & 64 & viscous oil \\
$\mathbf{6 1}$ & 87 & viscous oil & $\mathbf{9 l}$ & 96 & viscous oil \\
$\mathbf{6 m}$ & 81 & viscous oil & $\mathbf{9 m}$ & 71 & viscous oil \\
$\mathbf{6 n}$ & 91 & $70.6 \sim 71.2$ & $\mathbf{9 n}$ & 68 & viscous oil \\
$\mathbf{6 0}$ & 90 & $66.7 \sim 67.7$ & $\mathbf{9 0}$ & 67 & viscous oil \\
$\mathbf{6 p}$ & 91 & $131.4 \sim 132.2$ & & & \\
\hline
\end{tabular}


Table S2. Herbicidal activity of cis-p-menthane bis-5-bromo-2-furyl-amines 6k against shoot growth of barnyardgrass.

\begin{tabular}{|c|c|c|c|c|c|c|c|c|c|c|c|c|c|c|c|c|c|c|c|c|c|c|c|c|c|c|c|c|c|c|c|c|c|}
\hline \multirow{3}{*}{$\frac{c(\mathrm{mmol} / \mathrm{L})}{2.5}$} & \multicolumn{15}{|c|}{$\begin{array}{c}\text { germination } \\
\text { number }\end{array}$} & \multicolumn{18}{|c|}{ The length of shoots (mm) } \\
\hline & \multirow{2}{*}{$\frac{1}{6}$} & \multirow{2}{*}{$\frac{2}{9}$} & \multirow{2}{*}{$\frac{3}{2}$} & \multicolumn{10}{|c|}{ Parallel 1} & \multicolumn{10}{|c|}{ Parallel 2} & \multicolumn{10}{|c|}{ Parallel 3} \\
\hline & & & & 1 & 8 & 10 & 16 & 6 & 15 & 0 & 0 & 0 & 0 & 14 & 8 & 17 & 16 & 5 & 16 & 15 & 2 & 16 & 0 & 18 & 9 & 0 & 0 & 0 & 0 & 0 & 0 & 0 & 0 \\
\hline 1.25 & 10 & 1 & 5 & 18 & 18 & 7 & 18 & 25 & 20 & 21 & 21 & 18 & 25 & 17 & 0 & 0 & 0 & 0 & 0 & 0 & 0 & 0 & 0 & 13 & 12 & 12 & 5 & 3 & 0 & 0 & 0 & 0 & 0 \\
\hline 0.63 & 10 & 10 & 10 & 29 & 19 & 23 & 25 & 18 & 28 & 20 & 33 & 32 & 22 & 22 & 33 & 19 & 15 & 10 & 17 & 33 & 21 & 22 & 20 & 32 & 32 & 21 & 19 & 29 & 21 & 16 & 23 & 17 & 25 \\
\hline 0.31 & 10 & 10 & 9 & 34 & 33 & 18 & 23 & 19 & 18 & 13 & 17 & 28 & 28 & 35 & 27 & 21 & 23 & 18 & 23 & 19 & 15 & 23 & 4 & 28 & 32 & 23 & 32 & 19 & 23 & 33 & 34 & 24 & 0 \\
\hline 0.16 & 8 & 8 & 8 & 35 & 41 & 34 & 25 & 26 & 23 & 24 & 20 & 0 & 0 & 38 & 29 & 23 & 27 & 27 & 15 & 26 & 27 & 0 & 0 & 24 & 31 & 33 & 35 & 29 & 41 & 36 & 32 & 0 & 0 \\
\hline 0.08 & 10 & 10 & 10 & 22 & 23 & 35 & 32 & 32 & 24 & 25 & 26 & 32 & 23 & 26 & 25 & 33 & 31 & 37 & 26 & 30 & 29 & 21 & 36 & 35 & 27 & 23 & 22 & 26 & 21 & 37 & 39 & 33 & 36 \\
\hline Blank & 10 & 9 & 10 & 31 & 15 & 39 & 35 & 37 & 31 & 49 & 44 & 38 & 35 & 41 & 31 & 41 & 42 & 33 & 38 & 27 & 32 & 31 & 28 & 39 & 28 & 35 & 38 & 41 & 36 & 41 & 42 & 44 & 49 \\
\hline
\end{tabular}

Notes: ${ }^{\mathrm{a}}$ The germination number refers to the greatest common divisor of the seeds that germinated in roots or stems.

Table S3. Herbicidal activity of cis-p-menthane bis-5-bromo-2-furyl-amines 6k against root growth of barnyardgrass.

\begin{tabular}{|c|c|c|c|c|c|c|c|c|c|c|c|c|c|c|c|c|c|c|c|c|c|c|c|c|c|c|c|c|c|c|c|c|c|}
\hline \multirow{3}{*}{$\frac{c(\mathrm{mmol} / \mathrm{L})}{1.25}$} & \multicolumn{15}{|c|}{$\begin{array}{l}\text { germination } \\
\text { number }\end{array}$} & \multicolumn{18}{|c|}{ The length of roots (mm) } \\
\hline & \multirow{2}{*}{$\frac{1}{10}$} & \multirow{2}{*}{$\frac{2}{1}$} & \multirow{2}{*}{$\frac{3}{5}$} & \multicolumn{10}{|c|}{ Parallel 1} & \multicolumn{10}{|c|}{ Parallel 2} & \multicolumn{10}{|c|}{ Parallel 3} \\
\hline & & & & 0 & 0 & 0 & 0 & 0 & 0 & 0 & 0 & 0 & 0 & 0 & 0 & 0 & 0 & 0 & 0 & 0 & 0 & 0 & 0 & 0 & 0 & 0 & 0 & 0 & 0 & 0 & 0 & 0 & 0 \\
\hline 0.63 & 10 & 10 & 10 & 5 & 10 & 25 & 12 & 10 & 6 & 23 & 8 & 10 & 13 & 15 & 2 & 0 & 8 & 15 & 2 & 22 & 4 & 15 & 8 & 12 & 15 & 10 & 12 & 6 & 22 & 9 & 7 & 2 & 0 \\
\hline 0.31 & 10 & 10 & 9 & 15 & 10 & 9 & 5 & 20 & 5 & 12 & 19 & 30 & 25 & 37 & 28 & 28 & 29 & 20 & 26 & 23 & 27 & 29 & 23 & 25 & 10 & 30 & 10 & 23 & 30 & 27 & 21 & 27 & 0 \\
\hline 0.16 & 8 & 8 & 8 & 15 & 20 & 25 & 19 & 11 & 19 & 25 & 26 & 0 & 0 & 25 & 22 & 36 & 32 & 45 & 8 & 18 & 33 & 0 & 0 & 26 & 14 & 36 & 30 & 10 & 16 & 22 & 17 & 0 & 0 \\
\hline 0.08 & 10 & 10 & 10 & 37 & 12 & 30 & 27 & 31 & 27 & 45 & 28 & 35 & 28 & 35 & 25 & 31 & 19 & 48 & 25 & 21 & 49 & 25 & 39 & 29 & 40 & 20 & 31 & 49 & 27 & 33 & 45 & 21 & 39 \\
\hline Blank & 10 & 9 & 10 & 35 & 21 & 35 & 28 & 47 & 32 & 31 & 29 & 34 & 35 & 35 & 22 & 32 & 45 & 61 & 27 & 30 & 40 & 32 & 35 & 41 & 31 & 31 & 42 & 37 & 31 & 49 & 37 & 28 & 36 \\
\hline
\end{tabular}


Table S4. Inhibition efficacy of aromatic groups substituted $p$-menthene type secondary amines $9 \mathbf{a} \sim 9 \mathbf{j}$ against shoot growth of barnyardgrass.

\begin{tabular}{ccccccccccc}
\hline Compd. & 10.00 a & 5.00 & 2.50 & 1.25 & 0.63 & 0.31 & 0.16 & 0.08 & 0.04 & 0.02 \\
\hline $\mathbf{9 a}$ & $/$ & $/ \mathrm{b}$ & 41.5 & 33.7 & 35.8 & 30.6 & 6.9 & $-\mathrm{c}$ & $/$ & $/$ \\
$\mathbf{9 b}$ & $/ \mathrm{b}$ & $-\mathrm{d}$ & 41.5 & 38.8 & 24.1 & 20.2 & 24.7 & 8.6 & $-^{\mathrm{c}}$ & $/$ \\
$\mathbf{9 c}$ & $/$ & $/ \mathrm{b}$ & 32.1 & 22.3 & 19.1 & 13.1 & $-\mathrm{c}$ & $/$ & $/$ & $/$ \\
$\mathbf{9 d}$ & $/ \mathrm{b}$ & $-\mathrm{d}$ & 60.3 & 54.3 & 39.8 & 42.9 & 26.2 & 9.1 & $-\mathrm{c}$ & $/$ \\
$\mathbf{9 e}$ & $-\mathrm{d}$ & 20.3 & 11.3 & 6.6 & 3.0 & $-\mathrm{c}$ & $/$ & $/$ & $/$ & $/$ \\
$\mathbf{9 f}$ & $-\mathrm{d}$ & 24.3 & 17.0 & 8.5 & 4.5 & $-\mathrm{c}$ & $/$ & $/$ & $/$ & $/$ \\
$\mathbf{9 g}$ & $/ \mathrm{b}$ & $-\mathrm{d}$ & 32.1 & 22.3 & 19.1 & 13.2 & $-\mathrm{c}$ & $/$ & $/$ & $/$ \\
$\mathbf{9 h}$ & $/ \mathrm{b}$ & $-\mathrm{d}$ & 28.9 & 23.4 & 17.6 & 11.5 & 7.2 & 6.8 & $-\mathrm{c}$ & $/$ \\
$\mathbf{9 i}$ & 47.4 & 26.5 & 11.3 & 7.4 & 5.4 & $-\mathrm{c}$ & $/$ & $/$ & $/$ & $/$ \\
$\mathbf{9 j}$ & $/ \mathrm{b}$ & $-\mathrm{d}$ & 39.5 & 33.7 & 22.5 & 18.7 & 12.0 & $-\mathrm{c}$ & $/$ & $/$ \\
\hline
\end{tabular}

Notes: ${ }^{\mathrm{a}}$ The data of this line are the concentration of different $p$-menthane type Schiff base derivatives solutions $(\mathrm{mmol} / \mathrm{L})$.

${ }^{\mathrm{b}}$ The data at and higher or lower than this concentration were not determined.

${ }^{\mathrm{c}}$ Have no inhibition activity at this concentration.

${ }^{\mathrm{d}}$ The samples could not absolutely dissolved at and higher than this concentration. 
Table S5. Toxicity regression equations, $\mathrm{IC}_{50}$ and $\mathrm{IC}_{90}$ of hydrogenated p-menthene type Schiff bases $9 \mathrm{a} \sim 9 \mathrm{q}$ against the shoot growth of barnyardgrass.

\begin{tabular}{|c|c|c|c|}
\hline Compd. & toxicity regression equation & $\mathrm{IC}_{50}(\mathrm{mmol} / \mathrm{L})$ & $\mathrm{IC}_{90}(\mathrm{mmol} / \mathrm{L})$ \\
\hline $9 \mathbf{a}$ & $\begin{array}{c}y=4.5800+0.8716 x \\
r=0.8194\end{array}$ & 3.0333 & $>20$ \\
\hline $9 b$ & $\begin{array}{c}y=4.5574+0.6725 x \\
r=0.9091\end{array}$ & 4.5519 & $>20$ \\
\hline $9 \mathrm{c}$ & $\begin{array}{c}y=4.2314+0.6918 x \\
r=0.9870\end{array}$ & 12.9159 & $>20$ \\
\hline 9d & $\begin{array}{c}y=5.0011+0.9619 x \\
r=0.9367\end{array}$ & 0.9974 & $>20$ \\
\hline $9 e$ & $\begin{array}{c}y=3.3597+1.1444 x \\
r=0.9989\end{array}$ & 27.1229 & $>20$ \\
\hline 9f & $\begin{array}{c}y=3.5398+1.1342 x \\
r=0.9963\end{array}$ & 19.3864 & $>20$ \\
\hline $9 \mathrm{~g}$ & $\begin{array}{c}y=4.2323+0.6871 x \\
r=0.9869\end{array}$ & 13.1021 & $>20$ \\
\hline $9 \mathrm{~h}$ & $\begin{array}{c}y=4.1798+0.6785 x \\
r=0.9877\end{array}$ & 16.1791 & $>20$ \\
\hline $9 \mathbf{i}$ & $\begin{array}{c}y=3.4925+1.2964 x \\
r=0.9683\end{array}$ & 14.5471 & $>20$ \\
\hline 9j & $\begin{array}{c}y=4.4537+0.7593 x \\
r=0.9924\end{array}$ & 5.2417 & $>20$ \\
\hline
\end{tabular}


Table S6. Inhibition efficacy of cis-p-menthane type Schiff bases against root growth of barnyardgrass.

\begin{tabular}{ccccccccccc}
\hline Compd. & $10.00^{\text {a }}$ & 5.00 & 2.50 & 1.25 & 0.63 & 0.31 & 0.16 & 0.08 & 0.04 & 0.02 \\
\hline $\mathbf{5 a}$ & $/$ & $/$ & $/ \mathrm{d}$ & 83.3 & 63.2 & 39.2 & 7.6 & $-^{\mathrm{c}}$ & $/^{\mathrm{b}}$ & $/$ \\
$\mathbf{5 b}$ & $/$ & $/$ & $/ \mathrm{b}$ & 100.0 & 96.2 & 91.4 & 41.8 & 12.2 & $-^{\mathrm{c}}$ & $/ \mathrm{b}$ \\
$\mathbf{5 c}$ & $/$ & $/$ & $/ \mathrm{b}$ & 100.0 & 99.0 & 80.4 & 51.9 & 27.2 & 18.1 & $-^{\mathrm{c}}$ \\
$\mathbf{5 d}$ & $/$ & $/ \mathrm{b}$ & $/ \mathrm{d}$ & 73.1 & 50.4 & 41.8 & 38.8 & 34.7 & $-^{\mathrm{c}}$ & $/ \mathrm{b}$ \\
$\mathbf{5 e}$ & $/$ & $/ \mathrm{b}$ & $/ \mathrm{d}$ & 86.4 & 83.5 & 56.9 & 44.9 & 12.6 & $-\mathrm{c}$ & $/ \mathrm{b}$ \\
$\mathbf{5 f}$ & $/$ & $/$ & $/ \mathrm{b}$ & 100.0 & 95.4 & 75.4 & 39.5 & 30.0 & 20.2 & 14.3 \\
$\mathbf{5 g}$ & $/$ & $/$ & $/ \mathrm{b}$ & 100.0 & 88.9 & 63.1 & 52.8 & 25.1 & 21.4 & $-\mathrm{c}$ \\
$\mathbf{5 h}$ & $/$ & $/$ & $/ \mathrm{b}$ & 100.0 & 95.4 & 75.4 & 39.5 & 30.0 & 20.2 & 14.3 \\
$\mathbf{5 i}$ & $/$ & $/ \mathrm{b}$ & $/ \mathrm{d}$ & 81.9 & 47.3 & 21.4 & 11.7 & $-\mathrm{c}$ & $/ \mathrm{b}$ & $/$ \\
$\mathbf{5 j}$ & $/ \mathrm{b}$ & $/ \mathrm{d}$ & 86.9 & 49.0 & 12.6 & 8.8 & $-\mathrm{c}$ & $/ \mathrm{b}$ & $/$ & $/$ \\
$\mathbf{5 k}$ & $/$ & $/$ & $/ \mathrm{b}$ & 100 & 91.0 & 84.1 & 69.7 & 61.2 & 44.2 & $-\mathrm{c}$ \\
$\mathbf{5 l}$ & $/$ & $/ \mathrm{d}$ & 88.0 & 77.2 & 62.8 & 58.4 & 40.9 & 45.4 & 24.3 & $-\mathrm{c}$ \\
$\mathbf{5 m}$ & $/ \mathrm{b}$ & 100 & 97.6 & 84.7 & 57.0 & 31.4 & 17.5 & $-\mathrm{c}$ & $/ \mathrm{b}$ & $/$ \\
$\mathbf{5 n}$ & $/ \mathrm{b}$ & 100 & 98.3 & 84.9 & 74.1 & 69.6 & 51.0 & 9.4 & $-\mathrm{c}$ & $/ \mathrm{b}$ \\
$\mathbf{5 0}$ & $/ \mathrm{d}$ & 93.3 & 87.5 & 61.4 & 5.5 & $-\mathrm{c}$ & $/ \mathrm{b}$ & $/$ & $/$ & $/$ \\
$\mathbf{5 p}$ & $/$ & 100 & 90.8 & 73.1 & 54.8 & 14.0 & 11.6 & $-\mathrm{c}$ & $/ \mathrm{b}$ & $/$ \\
\hline
\end{tabular}

Notes: ${ }^{\mathrm{a}}$ The data of this line are the concentration of different $p$-menthane type Schiff base derivatives solutions $(\mathrm{mmol} / \mathrm{L})$.

${ }^{\mathrm{b}}$ The data at and higher or lower than this concentration were not determined.

${ }^{c}$ Have no inhibition activity at this concentration.

${ }^{d}$ The samples could not absolutely dissolved at and higher than this concentration. 
Table S7. Toxicity regression equations, $\mathrm{IC}_{50}$ and $\mathrm{IC}_{90}$ of hydrogenated $p$-menthane type Schiff bases $3 a \sim 3 q$ against the root growth of barnyardgrass.

\begin{tabular}{|c|c|c|c|}
\hline Compd. & toxicity regression equation & $\mathrm{IC}_{50}(\mathrm{mmol} / \mathrm{L})$ & $\mathrm{IC}_{90}(\mathrm{mmol} / \mathrm{L})$ \\
\hline $5 \mathbf{a}$ & $\begin{array}{c}y=5.8193+2.5954 x \\
r=0.9862\end{array}$ & 0.4834 & 1.5070 \\
\hline $5 \mathbf{b}$ & $\begin{array}{c}y=7.7000+3.4452 x \\
r=0.9787\end{array}$ & 0.1646 & 0.4940 \\
\hline $5 c$ & $\begin{array}{c}y=7.4721+2.6401 x \\
r=0.9653\end{array}$ & 0.1158 & 0.3540 \\
\hline $5 d$ & $\begin{array}{c}y=5.3357+0.7674 x \\
r=0.9111\end{array}$ & 0.3652 & 17.0851 \\
\hline $5 e$ & $\begin{array}{c}y=6.1337+1.8568 x \\
r=0.9696\end{array}$ & 0.2451 & 1.2013 \\
\hline $5 f$ & $\begin{array}{c}y=6.2048+1.1528 x \\
r=0.9574\end{array}$ & 0.0901 & 1.1657 \\
\hline $5 g$ & $\begin{array}{c}y=6.3790+1.6709 x \\
r=0.9721\end{array}$ & 0.1495 & 0.8743 \\
\hline $5 \mathrm{~h}$ & $\begin{array}{c}y=6.6319+1.7621 x \\
r=0.9495\end{array}$ & 0.1185 & 0.6326 \\
\hline $5 \mathbf{i}$ & $\begin{array}{c}y=5.5439+2.3367 x \\
r=0.9831\end{array}$ & 0.5851 & 2.0686 \\
\hline $5 \mathbf{j}$ & $\begin{array}{c}y=4.8007+2.8399 x \\
r=0.9661\end{array}$ & 1.1754 & 3.3224 \\
\hline $5 \mathbf{k}$ & $\begin{array}{c}y=6.5872+1.2254 x \\
r=0.9961\end{array}$ & 0.0507 & 1.1144 \\
\hline 51 & $\begin{array}{c}y=5.6762+0.9378 x \\
r=0.9725\end{array}$ & 0.1901 & 4.4205 \\
\hline $5 \mathrm{~m}$ & $\begin{array}{c}y=5.8517+2.4391 x \\
r=0.9904\end{array}$ & 0.4475 & 1.5005 \\
\hline $5 n$ & $\begin{array}{c}y=6.1881+1.9305 x \\
r=0.9567\end{array}$ & 0.2424 & 1.1179 \\
\hline 50 & $\begin{array}{c}y=4.5006+3.3713 x \\
r=0.9459\end{array}$ & 1.4065 & 3.3749 \\
\hline $5 p$ & $\begin{array}{c}y=5.4158+2.2406 x \\
r=0.9788\end{array}$ & 0.6523 & 2.4344 \\
\hline
\end{tabular}

